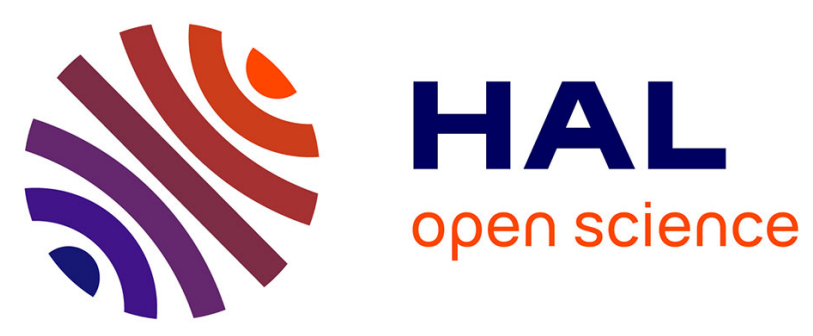

\title{
Contribution of a joint Bayesian inversion of VLBI and gravimetric data to the estimation of the free inner core nutation and free core nutation resonance parameters
}

Yann Ziegler, Sébastien Lambert, Ibnu Nurul Huda, Christian Bizouard, Séverine Rosat

\section{To cite this version:}

Yann Ziegler, Sébastien Lambert, Ibnu Nurul Huda, Christian Bizouard, Séverine Rosat. Contribution of a joint Bayesian inversion of VLBI and gravimetric data to the estimation of the free inner core nutation and free core nutation resonance parameters. Geophysical Journal International, 2020, 222 (2), pp.845-860. 10.1093/gji/ggaa181 . hal-02889603

\section{HAL Id: hal-02889603 https://hal.science/hal-02889603}

Submitted on 4 Jul 2020

HAL is a multi-disciplinary open access archive for the deposit and dissemination of scientific research documents, whether they are published or not. The documents may come from teaching and research institutions in France or abroad, or from public or private research centers.
L'archive ouverte pluridisciplinaire HAL, est destinée au dépôt et à la diffusion de documents scientifiques de niveau recherche, publiés ou non, émanant des établissements d'enseignement et de recherche français ou étrangers, des laboratoires publics ou privés. 


\title{
Contribution of a joint Bayesian inversion of VLBI and gravimetric data to the estimation of the free inner core nutation and free core nutation resonance parameters
}

\author{
Yann Ziegler, ${ }^{1}$ Sébastien B. Lambert, ${ }^{1}$ Ibnu Nurul Huda, ${ }^{1}$ Christian Bizouard ${ }^{1}$ and \\ Séverine Rosat ${ }^{\oplus 2}$ \\ ${ }^{1}$ Observatoire de Paris, Université PSL, CNRS, Sorbonne Université, LNE, Paris, France.E-mail: yann.ziegler@obspm.fr \\ ${ }^{2}$ Université de Strasbourg, CNRS, EOST, IPGS UMR 7516, Strasbourg, France
}

Accepted 2020 April 11. Received 2020 April 6; in original form 2019 May 15

\begin{abstract}
S U M MAR Y
The rotational motions of the internal Earth layers induce resonances in the Earth nutations and tidal gravimetric response to external luni-solar gravitational forcings. The characterization of these resonances is a mean of investigating the deep Earth properties since their amplitudes and frequencies depend on a few fundamental geophysical parameters. In this work, we focus on the determination of the free core nutation and free inner core nutation periods and quality factors from the Bayesian inversion of VLBI and gravimetric data. We make a joint inversion of data from both techniques and show that, even if the results are only slightly different from the inversion of VLBI data alone, such approach may be valuable in the future if the accuracy of gravimetric data increases. We also briefly discuss the polar motion resonance, which is related to the Chandler Wobble as seen from the diurnal frequency band. Our overall estimates of the FCN period and quality factor, $T_{\mathrm{FCN}}=(-430.2,-429.8)$ solar days and $Q_{\mathrm{FCN}}=(15700$, 16700), respectively, are in good agreement with other studies, albeit slightly different for unclear reasons. Despite some concerns about the detection and characterization of the FICN, it seems that we could also successfully estimate its period, $T_{\mathrm{FICN}}=(+600,+1300)$ solar days, and give a loose estimate of the upper bound on its quality factor.
\end{abstract}

Key words: Core; Earth rotation variations; Time variable gravity; Joint inversion.

\section{INTRODUCTION}

\subsection{Earth rotational eigenmodes, forced nutations and resonances}

The Earth internal structure and the external forcings exerted on it jointly determine the Earth rotational motions. The external forcings of astronomical origin are already well-known whereas some of the geophysical properties of the deep Earth remain largely undetermined. Studying the rotation of the Earth is thereby one of the few ways of filling this knowledge gap.

The three main layers of the solid Earth, namely the mantle, the fluid outer core and the solid inner core, rotate with some degrees of freedom relative to each others. Their rotation gives rise to four rotational eigenmodes which are the Chandler Wobble $(\mathrm{CW})$, the Free Core Nutation (FCN), the Free Inner Core Nutation (FICN) and the Inner Core Wobble (ICW). Along with the forced annual oscillation, the $\mathrm{CW}$ is the main component of the polar motion, the movement of the Earth rotation axis relative to the mantle; its period is close to 14 months in the Terrestrial rotating Reference Frame (TRF). The FCN and FICN are, respectively, retrograde and prograde nutational motions of the Earth rotation axis relative to the Celestial non-rotating Reference Frame (CRF). In the CRF, the FCN period is close to -430 mean solar days (with the convention 'negative retrograde periods') whereas the value of the FICN period is still debated (see Section 1.3). In the TRF, the FCN translates into a Nearly Diurnal Free Wobble (NDFW), a retrograde motion of the instantaneous rotation axis around the Earth figure axis. The NDFW has an amplitude about 430 times smaller than the FCN, following the ratio between the FCN and NDFW respective periods. Similarly, the FICN also translates into a nearly diurnal retrograde wobble in the TRF. The ICW, CW of the solid inner core, has yet to be irrefutably observed and we will have no concern with it in this work.

When forces are exerted on the Earth at frequencies close to its rotational eigenmodes frequencies, resonances appear. As demonstrated by Melchior \& Georis (1968), there are simple mathematical relations between the frequencies and amplitudes of tidal waves and nutations, as both are different aspects of the Earth response to the same luni-solar perturbing potential. Thus, we can theoretically observe the resonances due to the Earth internal layers in both nearly diurnal tidal waves (in the TRF) and long periodic nutations (in 
the CRF). For the same reason, the theoretical study of the FCN or NDFW is tightly related to Earth tides studies.

The periods and amplitudes of these resonances are closely related to the parameters of the rotational eigenmodes which themselves depend on a few fundamental geophysical parameters. The whole Earth, core-mantle boundary (CMB) and inner core-outer core boundary (ICB) flattenings, a set of compliance parameters and the magnetic or topographic couplings between the Earth layers are some examples. That is why the characterization of the resonances can be so insightful in global geodynamic studies.

Astrometric and geophysical observations provide time-series for the Earth nutations and solid Earth tides, respectively. In particular, the data used when studying the Earth rotational eigenmodes and resonances come mostly from two techniques: the very long baseline interferometry (VLBI) and surface gravimetry using superconducting gravimeters (SGs) worldwide. These techniques yield independent data sets which have been both used successfully to characterize the resonance associated with the FCN (Section 1.2). The joint inversion of VLBI and gravimetric data had yet to be done, however. This is one of the main contribution of this work.

The next sections of this introduction provide an overview of the key contributions in the study of the rotation of the Earth. We remind the distant origin of some questions that are still open to this day and emphasize some erroneous approximations or hasty interpretations that we should now take care to avoid. Then, we describe in Section 2 the theoretical framework we use in the present study. We define the frequencies, periods and quality factors appearing in the transfer functions for the Earth rotation axis motion and the tidal gravimetric factor, and discuss the key points of such a formalism. In Section 3, we briefly describe the main ideas behind the Bayesian inversion. The VLBI and gravimetric data are introduced in Section 4. The synthetic tests in Section 5 are an important part of this work. We rely on various inversions of synthetic data to empirically assess the reliability and accuracy of the Bayesian inversion. The results of the Bayesian inversion of VLBI and gravimetric data, either separately or jointly, are described in Section 6. Finally, we discuss the overall validity of our results and their limitations, especially regarding the FICN, in Section 7.

\subsection{Past theoretical developments and observations}

The pioneering studies on the influence of the fluid core on Earth rotational dynamics date back to the eighteenth century (Hopkins 1839; Hough 1895; Sludskii 1896; Poincaré 1910) but for the sake of brevity, we will focus on modern developments directly relevant to this work. For the reader interested in pre-1980s studies, Rochester (1973), Toomre (1974), Rochester et al. (1974) and Melchior (1980) offer insightful reviews of the theory and observing attempts of the $\mathrm{FCN}$ at that time and provide clarifications on its essence.

In 1980, the International Astronomical Union (IAU) was relying on the linear momentum description and normal mode expansion of Wahr (1981a, c) for the computation of the reference nutation model parameters. As an alternative nutation theory, Sasao et al. (1980) developed an approachable and flexible framework using the angular momentum description (similar to the Euler-Liouville equations). Many subsequent works have been built upon this theory, including the current nutation reference model (MHB2000 from Mathews et al. 2002) of the IAU and the International Union of Geodesy and Geophysics.

In parallel to these theoretical studies, many authors have attempted to observe and characterize the resonance associated with the FCN. One of the first recognized observation of the FCN in nutations amplitudes derived from VLBI analysis was made by Herring et al. (1986). Their observation was actually a modification to Wahr (1981b) reference values and an upper bound on the FCN amplitude, but it was the first attempt to do 'Geodesy by Radio Astronomy' and to probe the CMB with VLBI data (Gwinn et al. 1986). In gravimetry, one of the first attempts to detect the NDFW is the work of Lecolazet \& Steinmetz (1974) who used data from a gravimeter in Strasbourg (France). Using their own analytical model to invert tidal gravity data from six superconducting gravimeters in central Europe, Neuberg et al. (1987) independently estimated the FCN period and quality factor. They found a period in partial agreement with Herring et al. (1986) results but a much smaller quality factor, both having larger uncertainties as is usually the case for such analyses of gravity data. Overall, however, discrepancy still remained between observed nutations amplitudes and values predicted by the theories then available.

As a response to this disagreement, another major theoretical advance was done by Mathews et al. (1991a) who included in their model the influence of the solid inner core. This influence had also been studied by Busse (1970) but only in an attempt to explain some features of the Chandler wobble. Mathews et al. (1991a) computations revealed two new eigenfrequencies due to the inner core, one of them being associated with a prograde nutation, the so-called FICN. Other authors also developed analytical theories for the free and forced motions of a rotating earth model including a solid inner core (de Vries \& Wahr 1991; Dehant et al. 1993; Legros et al. 1993) but in the 1990s, all theories yielded a FICN period smaller than $500 \mathrm{~d}$, which is almost half the current reference value.

From the 2000s onward, new studies examined the possible influence of previously neglected mechanisms, such as couplings between the outer core and inner core. Greff-Lefftz et al. (2000) could theoretically explain an FICN period of up to several thousands days involving viscomagnetic couplings at the ICB. Mathews (2001) computed a FICN period of 939 d using a modified PREM model without couplings. Refining the theory of Mathews et al. (1991a), the MHB2000 model of Mathews et al. (2002) is one of the last major development in the field. It notably includes the ocean tide effects, mantle anelasticity and electromagnetic couplings at the fluid core boundaries. Complementing these improvements in nutations theory, longer time series and refined data processing have since permitted more accurate estimates of the FCN resonance parameters, in better and better agreement, from either VLBI or gravity data. See Rosat et al. (2009, Table 1) for a detailed list of contemporary and older estimates from both techniques; more recent works are Koot et al. (2010), Rosat et al. (2017) and Zhu et al. (2017).

\subsection{Uncertainty on the FICN parameters}

The FCN period and quality factor seem now to be fairly well determined; the FICN parameters are still largely uncertain, however. This uncertainty is likely due to the weakness of the resonance associated with the FICN, relative to the amplitude of the uncorrected errors, making it especially difficult to detect and, a fortiori, to properly characterize. In the following paragraphs, we focus on the work of Mathews et al. (2002) that we will routinely write 'MHB' (for Mathews, Herring and Buffett). We also use their notations, except for the tildes which highlight the complex parameters and functions in the frequency domain. 
Table 1. Periods and quality factors of the PM, FCN and FICN resonances from the inversion of error-free or noisy synthetic data generated using MHB2000 model whose parameters are given in the last column. The number of significant figures is roughly chosen in such a way that the last figure may vary by at most a few units from one inversion to another with a different seed for the random sampling. This rule does not apply to the extrema values (inversions with noise). Periods are given in solar days in the TRF (PM resonance) or CRF (FCN, FICN). The scaling refers to the scaling factor applied to the data covariance matrix; its square root is the correcting factor applied to the uncertainty of the data. RMD means Relative Median Absolute Deviation, see eq. (15); $\Delta \epsilon$ is defined in eq. (16). wRMSE is the weighted Root Mean Squared Error (with the inverse of data uncertainty as weight), that is the residues.

\begin{tabular}{|c|c|c|c|c|c|}
\hline \multirow[t]{2}{*}{ Inverted parameters } & \multicolumn{2}{|c|}{ Inversion of synthetic error-free data } & \multicolumn{2}{|c|}{$\begin{array}{c}\text { Synthesis of valid inversions of data with realistic } \\
\text { noise level }\end{array}$} & \multirow{2}{*}{$\begin{array}{c}\text { MHB } \\
2000 \\
\text { (target) }\end{array}$} \\
\hline & 68 per cent $\mathrm{CIs}$ & RMD (per cent) & Extrema of the CIs & Extrema of $\Delta \epsilon$ & \\
\hline$Q_{1}$ & $(-9.66,-9.46)$ & 1 & -9.85 to -9.32 & -2.1 to +1.8 & -9.55 \\
\hline$T_{\mathrm{FCN}}$ & $(-430.24,-430.14)$ & 0.02 & -430.37 to -429.78 & -1.1 to +2.0 & -430.21 \\
\hline$Q_{\mathrm{FCN}}$ & $(19800,20200)$ & 1 & 19400 to 20700 & -2.1 to +2.0 & 20046 \\
\hline$(\text { scaling })^{1 / 2}$ & \multirow{2}{*}{\multicolumn{2}{|c|}{$\begin{array}{l}\text { not adjusted } \\
\quad \sim 10^{-6} \text { (theoretically null) }\end{array}$}} & \multirow{2}{*}{\multicolumn{2}{|c|}{$\begin{array}{l}0.84 \text { to } 1.3 \\
\quad \text { between } 3.6 \cdot 10^{-4} \text { and } 8.6 \cdot 10^{-4}\end{array}$}} & \\
\hline wRMSE & & & & & \\
\hline
\end{tabular}

MHB estimated a period of 1030 solar days for the FICN, with acceptable values ranging from 840 to $1310 \mathrm{~d}$, as shown by Dehant et al. (2005). This value has been the standard ever since. Nevertheless, the model parametrization of MHB induces a strong correlation between some of the chosen geophysical parameters, as Rosat et al. (2017) have shown. These correlations are only partly mitigated by using default, non-adjusted values for some parameters but this approach has its own downsides.

Using MHB notations, the FICN frequency is

$\tilde{\sigma}_{3}=\left(\alpha_{2} e_{s}+\tilde{v}\right)-\tilde{K}^{\mathrm{ICB}}-1$,

with $\tilde{K}^{\mathrm{ICB}}$ the electromagnetic coupling constant at the ICB and $\left(\alpha_{2} e_{s}+\tilde{v}\right)$ one of the so-called basic earth parameter, with $e_{s}$ the ICB ellipticity, $\tilde{v}$ one of the model compliances and $\alpha_{2}$ a parameter quantifying the effect of the torques on the inner core (Mathews et al. 1991a). The only parameter inverted by MHB in eq. (1) is $\tilde{K}^{\mathrm{ICB}}$. Thereby, their estimation of the FICN frequency is entirely determined by the estimation of this coupling constant.

The coupling constant $\tilde{K}^{\mathrm{ICB}}$ also appears in the FCN frequency, which is

$\tilde{\sigma}_{2}=-\frac{A}{A_{m}}\left(e_{f}-\tilde{\beta}+\tilde{K}^{\mathrm{CMB}}+\frac{A_{s}}{A_{f}} \tilde{K}^{\mathrm{ICB}}\right)-1$,

with $A, A_{m}, A_{f}$ and $A_{s}$ the whole Earth, mantle, outer core and inner core mean equatorial moments of inertia, respectively, and $\tilde{K}^{\mathrm{CMB}}$ the electromagnetic coupling constant at the CMB. Using Mathews et al. (2002, 1991b) estimates for all the parameters in eqs (1) and (2), we can readily check that $\tilde{K}^{\mathrm{ICB}}$ alone accounts for a little bit less than 1 per cent of the value of $\tilde{\sigma}_{2}+1$ but could explain up to a third of $\tilde{\sigma}_{3}+1$. The relative uncertainty on the FCN frequency estimate is at least a hundred times smaller than the relative uncertainty on the FICN frequency, however (Mathews et al. 2002, table $3 a)$. Thus, the determination of the FICN frequency via $K^{\mathrm{CMB}}$, could be, in fact, mostly relying on the FCN frequency estimate, without any clear-cut observation of the FICN resonance. In other words, the value of $\tilde{K}^{\mathrm{ICB}}$ — which fully determined the FICN frequency in MHB formalism - could result from the observation of either rotation mode, the relative importance of each in the estimation being unclear. The correlation between $\tilde{K}^{\mathrm{ICB}}$ and $\tilde{K}^{\mathrm{CMB}}$ may even worsen the situation.

From a geophysical point of view, the estimation of $\tilde{K}^{\mathrm{ICB}}$ alone to determine the FICN frequency can also strongly bias the interpretation of the results. As MHB acknowledged themselves, any mistake in the value of $\left(\alpha_{2} e_{s}+\tilde{v}\right)$ would equivalently impact the estimated value of the coupling at the ICB, and reciprocally. Of course, adding new terms in the expression of $\tilde{\sigma}_{3}$ to account for other physical processes would increase the correlations between the inverted parameters, but not doing so implies a potentially significant underestimation of the uncertainty on $\tilde{K}^{\mathrm{ICB}}$. Buffett et al. (2002) extensively discussed the electromagnetic coupling and the plausibility of MHB results but, for example, they did not consider viscous or topographic couplings in their interpretation (they did consider inner core viscosity though). Koot et al. (2010), coming to different conclusions regarding the coupling at the ICB, acknowledged that it is still very uncertain to distinguish electromagnetic coupling from other couplings or even from other internal processes. Successive studies on the topic have either largely used MHB FICN parameters values (Koot et al. 2008), or have produced results in quite varying agreement (Rosat et al. 2017). In some recent investigations, the FICN was not even reliably detected at all (Lambert et al. 2012; Rosat et al. 2016; Zhu et al. 2017). Such lack of reproducibility in FICN observations suggests that separating the ambiguous contributions of different physical processes on the inner core rotation might present a long-lasting challenge.

\section{NUTATIONAL AND TIDAL EARTH RESPONSE}

\subsection{Definitions}

Following a widespread convention, we will denote $\sigma$ the frequency in cycles per sidereal day (cpsd) as counted in the TRF, with retrograde and prograde motions having negative and positive frequencies, respectively. In the $\mathrm{CRF}$, this convention translates to $\sigma<-1$ for retrograde motions and $\sigma>-1$ for prograde motions. Thus, the FCN and FICN, which are long periodic motions as seen from the $\mathrm{CRF}$, have frequencies with real parts close to $\sigma=-1$ in the TRF whereas $\sigma \gtrsim 0$ for the polar motion.

In contrast with the generic real-valued frequency $\sigma$, we will denote $\tilde{\sigma}$ the complex-valued frequency in cpsd of any (pseudo)periodic damped motion as seen from the TRF. This frequency has a non-zero imaginary part to express the attenuation of the oscillations due to various dissipative processes. Following MHB, we will append to $\tilde{\sigma}$ an index ranging from 1 to 4 to identify the rotational eigenmode to which the frequency corresponds: 1 for the polar motion (PM) resonance, 2 for the FCN, 3 for the FICN and 4 
for the ICW (the modes are sorted in order of resonance amplitude). The index $\alpha$ in $\tilde{\sigma}_{\alpha}$ will routinely refer to any of the four resonances.

Note that the resonance frequencies do not necessarily match the eigenfrequencies, which is why we consider the PM resonance instead of the usual CW. As discussed by MHB, the resonance frequencies of a system whose parameters are frequency dependent will generally differ from the system eigenfrequencies. This is especially relevant for the PM resonance, which is the distant manifestation of the $\mathrm{CW}$ in the diurnal frequency band. Indeed, the PM depends on compliances which are frequency dependent because of mantle anelasticity and ocean tides and whose excitation lies in a different frequency band than the mode itself. An extended discussion on this topic is provided in Bizouard et al. (2019). The eigenfrequencies will therefore be written $\tilde{\sigma}_{\mathrm{CW}}, \tilde{\sigma}_{\mathrm{FCN}}, \tilde{\sigma}_{\mathrm{FICN}}$ and $\tilde{\sigma}_{\text {ICW }}$ to distinguish them from the resonance frequencies. We have already noted that $\tilde{\sigma}_{1} \neq \tilde{\sigma}_{\mathrm{CW}}$. On the contrary, the excitations being in the same frequency band as the FCN and FICN, we can consider that $\tilde{\sigma}_{2}=\tilde{\sigma}_{\mathrm{FCN}}$ and $\tilde{\sigma}_{3}=\tilde{\sigma}_{\mathrm{FICN}}$.

For the FCN and FICN, the periods in mean solar days in the $\mathrm{CRF}$ are defined as

$$
T_{2,3}=\frac{1}{k\left(1+\Re \tilde{\sigma}_{2,3}\right)}
$$

whereas for the CW (and similarly for the ICW), the period is more usually given in mean solar days in the TRF as

$$
T_{1}=\frac{1}{k \Re \tilde{\sigma}_{1}}
$$

with $k$ the ratio between the mean solar day and the sidereal day, which is close to 1.0027379 and $\Re \tilde{\sigma}$ is the real part of $\tilde{\sigma}$.

The definition of the quality factor requires a short physical discussion first. The quality factor can be interpreted as half the number of oscillatory cycles occurring before the amplitude of a damped oscillation has dropped with a factor $e$ (here $e \approx 2.718$ is the natural basis of the logarithm). However, the duration of one oscillatory cycle depends on the reference system, which can be arbitrarily chosen. The meaningful physical quantity here is actually the time needed for the attenuation to significantly damped the oscillation; it is inversely proportional to the imaginary part of the frequency and, contrary to the real part, it is independent of the reference system. It might seem counter-intuitive at first that the quality factor of the Earth rotational eigenmodes depends on the reference system but this is probably due to the fact that most computations of its value have been provided in the TRF without stating this choice explicitly (Chao \& Hsieh (2015) is a notable exception with an interesting discussion on this topic). Since the attenuation is always of geophysical origin, in the present work we make the explicit choice to quantify it in the TRF. We thus define the quality factor as

$Q_{\alpha}=-\frac{\Re \tilde{\sigma}_{\alpha}}{2 \Im \tilde{\sigma}_{\alpha}}$

for all modes or resonances ( $\Im \tilde{\sigma}$ is the imaginary part of $\tilde{\sigma}$ ). The minus sign comes from the frequency sign convention; it is required to have a positively-defined quality factor. Indeed, the real part of the frequency can be either positive or negative depending on the chosen convention but the imaginary part must always be positive for the oscillation to be damped. Note that $\tilde{\sigma}_{\alpha}$ has to be replaced with $\tilde{\sigma}_{C W}$, not $\tilde{\sigma}_{1}$, if we want to compute the $\mathrm{CW}$ quality factor using eq. (5), instead of the PM resonance quality factor.

\subsection{Transfer function for the Earth rotation axis motion}

Historically and for practical reasons, the computation of the effect of the tidal potential on the nutations amplitudes is done first for a given (rigid) reference earth model without resonance. Then, the effects of the resonances are expressed in the frequency domain as a transfer function $\tilde{T}(\sigma)$ defined as the ratio between $\tilde{\eta}$, the nutations amplitudes of a realistic Earth, or observed nutations, and $\tilde{\eta}_{R}$, the theoretical nutations amplitudes of a rigid earth model [such as RDAN97 of Roosbeek \& Dehant (1998) or REN2000 of Souchay et al. (1999)]. Because the ratio between the nutations $\tilde{\eta}$ and the corresponding wobbles $\tilde{m}$ of any earth model does not depend on the composition of the model, the previous definition is also valid when replacing the word 'nutation' with 'wobble'. Therefore, the transfer function is

$\tilde{\mathrm{T}}(\sigma)=\frac{\tilde{\eta}(\sigma)}{\tilde{\eta}_{R}(\sigma)}=\frac{\tilde{m}(\sigma)}{\tilde{m}_{R}(\sigma)}$.

with the $R$ subscript referring to the rigid earth nutation or wobble. For the sake of simplicity, we will sometimes use the word 'nutations' to refer to $\tilde{\mathrm{T}}(\sigma)$, implying that we have already normalized the observed nutations amplitudes by the non-resonant rigid earth nutations amplitudes.

After linearization, the transfer function (6) can be written explicitly (Mathews et al. 1991a)

$\tilde{\mathrm{T}}(\sigma)=\frac{e-\sigma}{e}\left[\mathbf{M}^{-1}(\sigma) \mathbf{y}(\sigma)\right]_{1}$,

with $e$ the Earth dynamic ellipticity, and $\mathbf{M}$ and $\mathbf{y}$ a four-by-four matrix and four-line column vector, respectively, whose coefficients are dependent on a set of geophysical parameters and on $\sigma$ [Mathews et al. 1991a, eqs (26b) and (26c)]. The subscript 1 refers to the first component of the $\mathbf{M}^{-1} \mathbf{y}$ column vector.

Eq. (7) can be analytically expanded but, because $\mathbf{M}$ is a four-byfour matrix, it would yield a complex expression which is neither very informative nor practical to handle. For that reason, Mathews et al. (1991a) and Mathews et al. (2002) on the one hand and Dehant et al. $(1993,2005)$ on the other hand, have made similar approximations to reach notably simpler forms of the transfer function, referred to as 'resonance formulae'. MHB have shown that the resonance formula including the four rotational eigenmodes $(\mathrm{CW}$, FCN, FICN and ICW) can be written

$\tilde{\mathrm{T}}\left(\sigma ; e \mid e_{R}\right)=\frac{e_{R}-\sigma}{e_{R}+1} N_{0}\left(1+(1+\sigma) \sum_{\alpha=1}^{4} \frac{\tilde{N}_{\alpha}}{\sigma-\tilde{\sigma}_{\alpha}}\right)$,

where $\tilde{N}_{\alpha}$ and $\tilde{\sigma}_{\alpha}$ are, respectively, the amplitudes and frequencies of the resonance associated with each mode and $N_{0}$ is the ratio between the real Earth dynamic ellipticity $e$ and the ellipticity $e_{R}$ used in the rigid earth nutation model.

Eq. (8) has been fully analytically determined and we thus have an analytical (approximate) expression for all $\tilde{N}_{\alpha}$ and $\tilde{\sigma}_{\alpha}$, as function of some fundamental geophysical parameters that can be estimated from data inversion. Nevertheless, the correlation between those parameters (see Section 1.3) counterbalance their theoretical interest, which is why we focus on the more robust estimation of the meta-parameters $\tilde{N}_{\alpha}$ and $\tilde{\sigma}_{\alpha}$.

\subsection{Tidal gravimetric factor}

Similarly, the tidal gravimetric factor is the transfer function, in the frequency domain, between the gravity perturbation $\Delta g^{t}$ due to the tidal luni-solar potential and the gravity response $\Delta g$ of the Earth 
to this perturbation:

$\tilde{\delta}(\sigma)=\frac{\Delta \tilde{g}(\sigma)}{\Delta \tilde{g}^{t}(\sigma)}$.

There are actually some enlightening subtleties in the exact physical definition of the gravimetric factor that we shall not discussed here. We refer the interested reader to Dehant et al. (1999).

The gravimetric factor is fully given by, for example, Legros et al. (1993). Nevertheless, applying the same kind of simplifications leading to the resonances formulae (8), the gravimetric factor reduces to (Rosat et al. 2017)

$\tilde{\delta}(\sigma)=\delta_{\text {ref }}+\sum_{\alpha=2}^{3} \frac{\tilde{N}_{\alpha}^{g}}{\sigma-\tilde{\sigma}_{\alpha}^{g}}$

with $\delta_{\text {ref }}$ the gravimetric factor reference value, that is to say the gravimetric factor far from any resonance. Practically, it can be computed as the mean of the gravimetric factor for two tidal constituents at the lowest and highest frequency in the frequency band of interest, which is the diurnal frequency band here.

\subsection{Corrections to the transfer functions}

The transfer functions $\tilde{\mathrm{T}}(\sigma)$ and $\tilde{\delta}(\sigma)$ defined in eqs (6) and (9), respectively, are actually ambiguous, depending on what is effectively included in their respective numerators $\tilde{m}(\sigma)$ and $\Delta \tilde{g}(\sigma)$. When using the observed polar motion or tidal gravity variations, it is implicitly acknowledged that a certain number of standard corrections have been applied beforehand. Similarly, the analytical transfer functions, computed for a given earth model, only incorporate a tiny part of the whole Earth system complexity. A very important consequence is that any two transfer functions, either determined from theory or observations, cannot be compared in a straightforward way, without some specific corrections.

To make this point clear, from now on, we will only denote by $\tilde{\mathrm{T}}$ and $\tilde{\delta}$ the complete transfer functions for nutations/wobbles and gravity, respectively. By 'complete', we mean that any astronomical or geophysical phenomenon affecting the transfer function has been included in the computation (still limiting the developments to linear effects). Symbolically, the Earth response to luni-solar forcing as for nutations and polar motion can thus be written

$\tilde{\mathrm{T}}(\sigma)=\tilde{\mathrm{T}}_{\mathrm{MHB}}(\sigma)+$ additional terms,

where $\tilde{\mathrm{T}}_{\mathrm{MHB}}$ refers to $\mathrm{MHB}$ transfer function and the additional terms encompass all contributions not included in the analytical theory such as the geodesic nutation, non-linear terms (Mathews et al. 2002, table 7) but also topographic coupling at the CMB for example.

Furthermore, a single scalar transfer function is not enough to fully describe the observed nutations. Indeed, they are also directly affected by the atmosphere and oceans which are themselves only partly governed by the luni-solar forcing and not fully integrated in nutation models. Several works have shown the importance of these geophysical effects on the rotation of the Earth, whether it be on length of day variation or polar motion and nutation (Barnes et al. 1983; Brzeziński et al. 2002; Vondrák \& Ron 2014, 2017). The observed nutation (and similarly for the wobble) must therefore be written

$\tilde{\eta}^{\mathrm{obs}}(\sigma)=\tilde{\mathrm{T}}(\sigma) \tilde{\eta}_{R}(\sigma)+\tilde{\mathrm{T}}_{S}(\sigma) \tilde{\chi}(\sigma)$,

where $\tilde{\chi}$ is the effective atmospheric and oceanic excitation function and $\tilde{T}_{S}$ is the transfer function between the atmosphere and oceans and the nutation (the index $S$ stands for surface processes). The determination of $\tilde{\mathrm{T}}_{S}(\sigma) \chi(\sigma)$ and an assessment of its effect on resonance parameters estimates is made by Nurul Huda et al. (2019); we use their corrections in the present work. Note that under certain hypotheses, the oceanic contribution can also be directly included in $\tilde{\mathrm{T}}$ by adjusting the compliances values (Mathews et al. 2002).

Another term, say $\tilde{q}(\sigma)$, should be added in the right-hand side of eq. (12) to take into account the errors on $\tilde{\eta}^{\text {obs }}$ which can be due either to noise, imperfection in the VLBI processing or other small errors due to the incompleteness of $\tilde{\mathrm{T}}, \tilde{\mathrm{T}}_{S}$ and $\tilde{\chi}$ models. The resulting equation is finally

$\tilde{\eta}^{o b s}(\sigma)=\tilde{\mathrm{T}}(\sigma) \tilde{\eta}_{R}(\sigma)+\tilde{\mathrm{T}}_{S}(\sigma) \tilde{\chi}(\sigma)+\tilde{q}(\sigma)$.

By definition, $\tilde{q}(\sigma)$ is unknown. It should be smaller than the uncertainty on $\tilde{\eta}^{\text {obs }}$, the remaining part of the uncertainty being mostly due to the atmosphere and oceans. For that reason, $\tilde{\mathrm{T}}_{S}(\sigma) \tilde{\chi}(\sigma)$ can be either estimated and subtracted from the observed nutations before estimating $\tilde{\mathrm{T}}$ parameters, or it can be used to inflate the uncertainty on $\tilde{\eta}^{o b s}$ before the inversion. Of course, all of the above discussion is also valid for the observed polar motion $\tilde{m}^{o b s}(\sigma)$.

In gravimetry, the nature of the corrections that need to be applied to the observations $\Delta \tilde{g}^{o b s}$ is slightly different although the main idea is also to only retain the contribution of the inner Earth. The main difference comes from the fact that the perturbations in gravity measurements are local and routinely corrected for each station simultaneously to the estimation of the gravimetric factor (Section 4.2). On the contrary, the perturbation of the Earth rotation axis by superficial geophysical fluid layers is of global nature and computed accordingly (atmospheric and oceanic angular momentum, $\tilde{\chi}$ function).

\section{BAYESIAN INVERSION}

\subsection{Principle of the Bayesian inversion}

The details of the Bayesian inversion can be found in Florsch \& Hinderer (2000), Koot et al. (2008) or Rosat et al. (2017) but we briefly remind here some key points of this method. First of all, the fundamental idea behind the Bayesian techniques is to consider that data are fixed, deterministic, whereas model parameters are probabilistic. The use of a probability law to describe model parameters does not reflect an intrinsic ambiguity in the parameters values but rather our own uncertainty on their values, considering the data we have collected. In other words, Bayesian method is a way of computing the probability of a given model knowing the data. Consequently, we work with probability distributions, the results of the inversion being the model parameters posterior distributions, not necessarily Gaussian, rather than single numbers with associated uncertainties.

The uncertainties on the results computed from the final distributions are, strictly speaking, credible regions or credible intervals (CIs). Unlike confidence intervals in traditional inversion techniques, a credible region can be interpreted in the following manner: knowing the data, there is a probability $p$ that the real model is in the credible region computed for value $p$ (VanderPlas 2014); this is a straightforward way of interpreting the results of a Bayesian inversion. The inversion itself is not a trivial problem but it can be solved using different numerical techniques. Here we use the Markov chain Monte Carlo (MCMC) algorithm implemented in Python PyMC library (Patil et al. 2010) to efficiently sample the space of model parameters in a way which ensures that after enough 

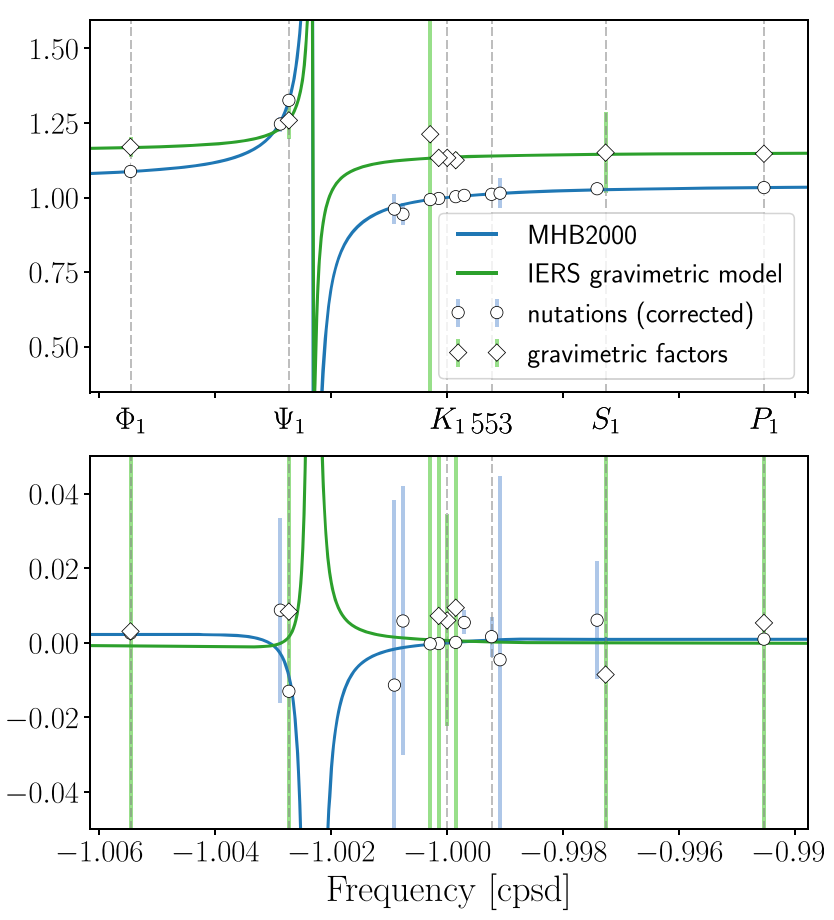

Figure 1. Real (top panel) and imaginary (bottom panel) part of the nutations (white dots) and tidal gravimetric factors (white diamonds) with their uncertainty in the diurnal frequency band in TRF. The corresponding transfer functions as defined in the IERS Conventions are in blue and green, respectively. The main tides are named and indicated by vertical dashed grey lines. Here, we limit the display to a narrow band around the FCN and FICN resonances but the data cover a larger frequency band, as illustrated by Fig. 3. For the sake of clarity, we also only show the nutations from the opa2018b data set and the gravimetric factors estimated from Strasbourg SGs measurements. See Fig. 3 for a plot of all the nutations inverted in this study; Fig. 8 similarly offers a more complete picture of the inverted gravimetric factors.

iterations, the extracted samples follow a distribution which is the sought-after posterior distribution for each of the model parameters.

The boundaries of the CIs we report in the next sections are given by the 0.16 and 0.84 quantiles, which means that 68 percent of the models sampled by the MCMC algorithm lie within this interval. Assuming Gaussian distributions for the posteriors, half of the CI span can thus be interpreted as the standard deviation of the estimate. To avoid such an assumption though, we compute instead the Median Absolute Deviation (MAD), scaled by the factor 1.4826 to match the standard deviation in the normal distribution case (Rousseeuw \& Croux 1993):

$\mathrm{MAD}=1.4826 \operatorname{median}\left(\left|X_{i}^{P}-\operatorname{median}\left(X^{P}\right)\right|\right)$,

with $X^{P}$ the set of all sampled values for the geophysical parameter $P$ and $X_{i}^{P}$ the elements of $X^{P}$. The MAD is then converted into Relative MAD (RMD) using the median of the estimates for normalization:

$\mathrm{RMD}=\frac{\mathrm{MAD}}{\left|\operatorname{median}\left(X^{P}\right)\right|}$.

This enables a straightforward comparison of the different levels of accuracy we can reach when inverting different synthetic or actual data sets.

\subsection{Errors and uncertainties}

Even though it is largely admitted that a decrease of the uncertainty on a given measurement or estimate is an improvement, it is actually much more desirable to improve the reliability of the uncertainties than to reduce the uncertainty itself. A reliable estimate of the uncertainty is a firm basis on which the observations and the theory can be confronted.

In both VLBI and gravimetric measurements, systematic errors are not fully corrected, either for theoretical or practical reasons. Our partial knowledge of radio-sources dynamics and the lack of a continuously updated, global atmospheric model to completely remove the atmospheric bias in VLBI delays are typical sources of systematic errors. The processing and analysis strategy is another source of systematic errors. Nutations amplitudes estimates from different analysis centres using almost the same initial data may differ from up to $10 \mu$ as, even when considering the long periodic nutations only. These discrepancies might not seem to be of much significance but, after normalization by the rigid earth nutations, they translate into up to a 2 per cent difference in the transfer function, which is sometimes larger than the uncertainty claimed by a single analysis centre. This is also large enough to influence the inversion of the FICN parameters as it weights the data, sometimes excessively, in favour of the largest nutations. There are also systematic differences, common to all analysis centres, that make VLBI data at odd with the resonance formula of Mathews et al. (2002). See Gattano et al. (2016) for an illustration of the importance of uncorrected errors in different VLBI analyses.

Such biases also exist in gravimetric measurements, especially because of local and regional gravitational perturbations which are difficult to properly model. For that reason, several authors have resorted to stacking (or other combinations methods) of gravimetric data to study the CW (Ziegler et al. 2016; Ding \& Chao 2017), FCN (Neuberg et al. 1987) or core undertones (Cummins et al. 1991).

Thus, we will take care in this work (1) not to underestimate the errors on the nutations amplitudes used in the inversion and (2) to thoroughly discuss the reliability of our uncertainties estimates before considering any geophysical interpretation.

The first point will be achieved by updating the estimated uncertainty on the nutations amplitudes during the inversion. Practically, we adjust a scaling factor for the data covariance matrix. The square root of this scaling factor is the factor by which data uncertainty is scaled.

The second point will be dealt with through a number of synthetic tests (Section 5) and by comparing the results from the inversion of the nutations data produced by different analysis centres ( $\mathrm{Sec}-$ tion 6.1). As highlighted in Section 3.1, the use of a Bayesian method will also be helpful in our quest for more reliable uncertainties estimates since it does not require additional computations but directly yields credible intervals instead, contrary to the least-square method for example.

\section{DATA}

The observed nutations and gravimetric factors can be thought of as samplings of the real-Earth transfer functions (6) and (9), assuming all corrections discussed in Section 2.4 have been applied or can be neglected. The number and quality of these samples are limited by the number of tidal frequencies at which the amplitude of the tidal potential is large enough to induce observable effects. Thus, only a few tens of nutations and tides, heterogeneously spread over the terrestrial diurnal frequency band, are actually large enough to be 


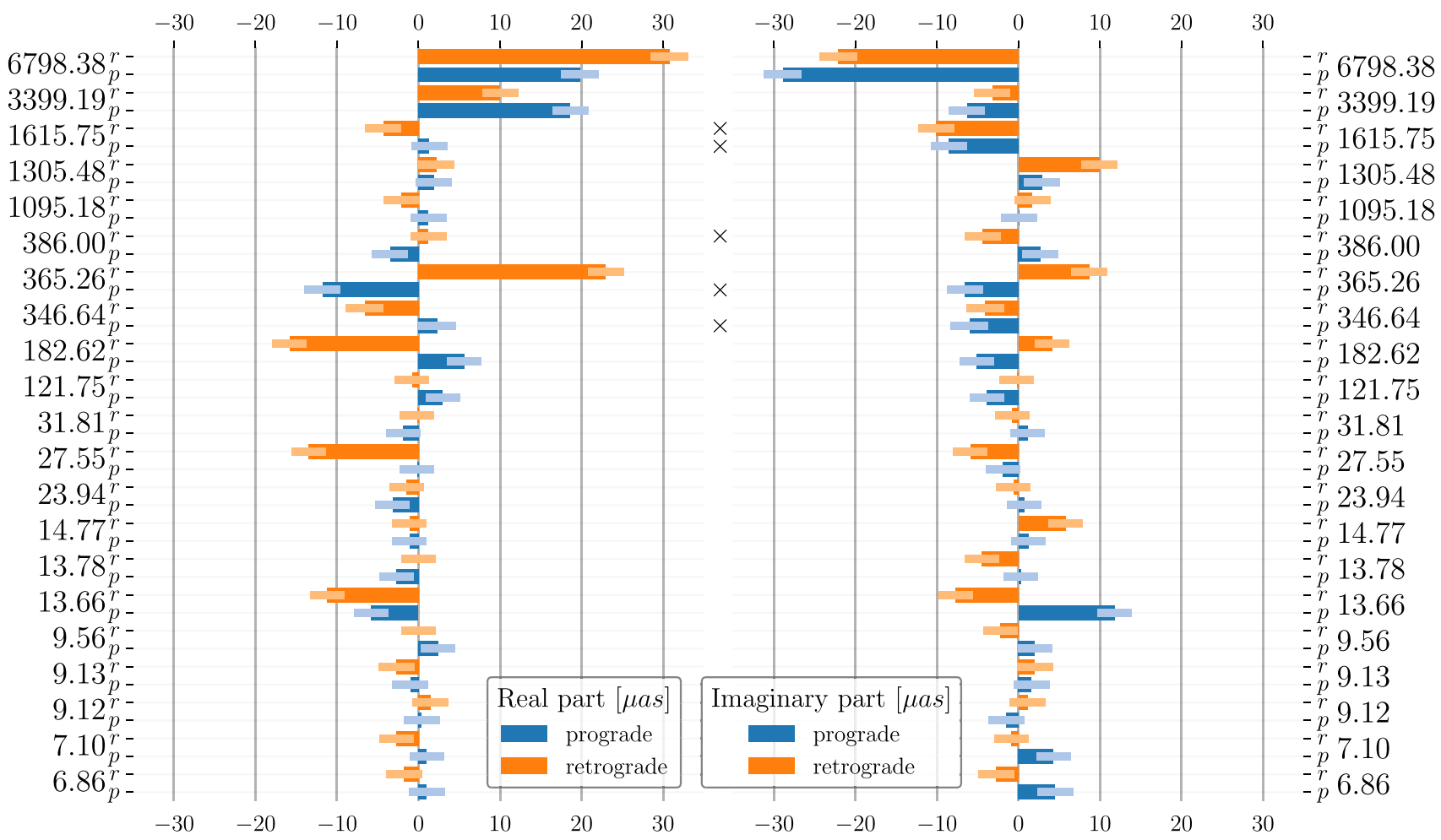

Figure 2. Real (left-hand side) and imaginary (right-hand side) part, in microarcseconds ( $\mu$ as), of the difference between the MHB nutations amplitudes and the nutations adjusted following the procedure described in Section 4.1 for series opa2018b. The nutations periods are in solar days and their prograde $(p)$ and retrograde $(r)$ components are depicted in blue and orange, respectively, along with the associated error which is always between 2.1 and $2.3 \mu$ as for all nutations. Note that we do not use the same convention as MHB in this work: here the real part is MHB's imaginary part and the imaginary part is the opposite of MHB's real part. The crosses at the middle of the plot indicate the nutations which are not included in the inversion of Section 6 (see Section 6.1 for a discussion about the exclusion of these data).

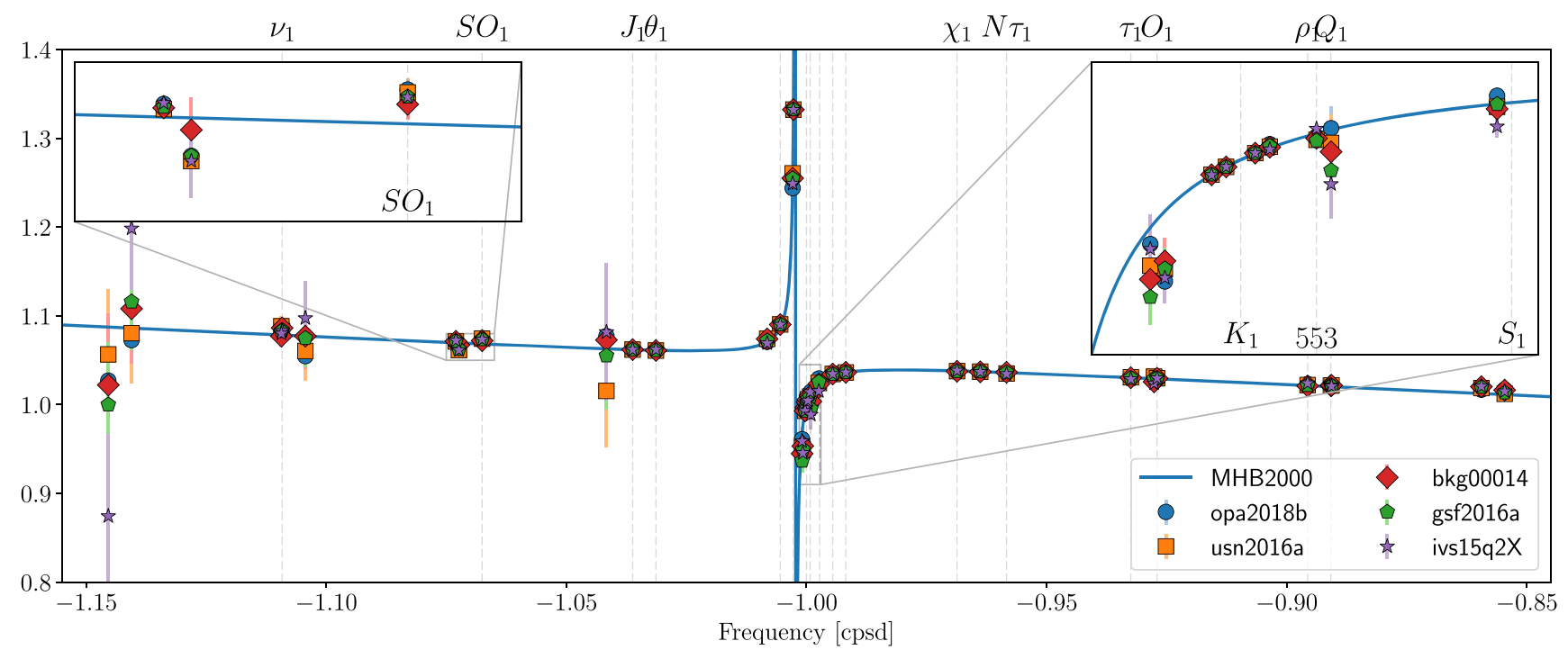

Figure 3. Real part of the nutations in the diurnal frequency band in TRF from five different analysis centres. The transfer functions MHB2000 as defined in the IERS Conventions is depicted in blue. The main tides are named and indicated by vertical dashed grey lines. Note the disagreement sometimes visible between different centres and, for some nutations, the systematic bias relative to the MHB2000 reference model as emphasized in the insets. For the sake of clarity, only the real part of the transfer functions and data is shown but similar comments can be made on the imaginary part.

observed and used to estimate the real-Earth nutational and gravimetric transfer functions. These data points are displayed on Fig. 1 with their error bars, on top of their respective reference transfer functions defined in the IERS Conventions (Petit \& Luzum 2010).

\subsection{VLBI analyses and corrections}

Our Bayesian inversion will be done on various sets of 21 prograde and retrograde nutation amplitudes adjusted in preliminary step on 
Table 2. Results of the Bayesian inversion of VLBI and gravimetric data, inverted either separately or jointly. As in Table 1, the number of significant figures is chosen to reflect the accuracy of the CIs given by the inversion, except for the RMDs which are always rounded toward infinity to ensure a conservative uncertainty estimation See Table 1 for a complete explanation on how to read this table. The CIs for the VLBI data are the union of the individual CIs obtained for each analysis centre, except for $T_{\mathrm{FICN}}$ where the spreading of the estimates would have overly inflate the CI (asterisk: opa2018b and ivs15q2X are excluded, see Fig. 7). Regarding $Q_{\mathrm{FICN}}$, NS stands for no stationarity, referring to the fact that the Markov chain failed to find a well-defined region of high probability for that parameter.

\begin{tabular}{|c|c|c|c|c|c|c|}
\hline Inverted parameters & \multicolumn{2}{|c|}{ VLBI data ( 5 centres) } & \multicolumn{2}{|c|}{ SGs data (9 stations) } & \multicolumn{2}{|c|}{ Joint inversion (opa2018b) } \\
\hline$T_{1}$ & $(380.4,382.1)$ & 0.15 & - & - & $(380.9,382.0)$ & 0.15 \\
\hline$T_{\mathrm{FCN}}$ & $(-430.2,-429.8)$ & 0.04 & $(-510,-410)$ & 10 & $(-430.1,-429.8)$ & 0.05 \\
\hline$Q_{\mathrm{FCN}}$ & $(15700,16700)$ & 3 & $(5800,29000)$ & 60 & $(15800,16100)$ & 1 \\
\hline$T_{\mathrm{FICN}}$ & $(+500,+1300)^{*}$ & 30 & $(+300,+1500)$ & 60 & $(+600,+1300)$ & 30 \\
\hline
\end{tabular}

nutation time-series derived from VLBI time delays. Time-series were computed by the various analysis centres from the International VLBI Service for Geodesy and Astrometry (IVS; Nothnagel et al. 2016) and released publicly via the IVS data centre. ${ }^{1}$ They consist of four individual series provided by the Budesamt fur Kartographie und Geodäsie (BKG, bkg00014), NASA Goddard Space Flight centre (GSFC, gsf2016a), Paris Observatory (opa2018b) and the US Naval Observatory (USNO, usn2016a) and the IVS combined series ivs15q2X obtained from the combination of normal equations of several IVS analysis centres. Though the various IVS analysis centres processed almost the same VLBI observational data base (about 10 millions delays since August 1979), they may have used some subtle variations in the analysis configuration or analysis software packages that result in small differences in the nutation time-series (e.g. excluding some sessions not originally designed for measuring the Earth orientation or regional networks, or different application of the minimal constraints to reference station or radio sources, modelling of the troposphere wet delay, gradients, and clock drift...). Although understanding the influence of these variations is beyond the scope of this study, the technical documentation about each solution can be found on the IVS data centre.

We adjusted the amplitudes of 21 nutation waves to the timeseries using a method similar to Gattano et al. (2016). For comparison purpose, we used the same nutation list as in Mathews et al. (2002). The differences between their nutations amplitudes and our adjusted amplitudes are displayed on Fig. 2 for (opa2018b). All amplitudes were adjusted by least-squares fit to the data after removing the free core nutation using a 7-yr sliding window. The original standard error was recalibrated iteratively similarly to Herring et al. (2002) in order that the chi-squared of the residuals be close to unity. The adjusted amplitudes (normalized by the rigid earth nutations amplitudes) are displayed on Fig. 3.

Regarding the atmospheric and oceanic effects discussed in Section 2.4, we then correct the nutations for both effects using the corrections given in Nurul Huda et al. (2019). We also inverted the uncorrected nutations for comparison.

\subsection{Gravimetric factors estimation}

The time-series from nine Superconducting Gravimeter sites (Bad Homburg, Cantley, Canberra, Membach, Metsahovi, Moxa, Strasbourg, Sutherland and Wettzell), with duration of 14-20 yr (from 1996-2001 to 2015-2016), were pre-processed to remove large earthquakes, to fill small gaps and glitches in a classical way (Hinderer et al. 2007) and decimated to $1 \mathrm{hr}$ in order to perform a tidal analysis. Superconducting Gravimeter Level 1 data products were downloaded from IGETS ${ }^{2}$ (Boy et al. 2020). The new version of the ETERNA3.4 software developed by (Wenzel 1996), ET34-ANA-V60, improved by Schüller (2015), was used. This latest upgrade enables to analyse non-linear tidal components as well as tidal constituents of the $3 \mathrm{rd}$ and 4 th degree in the tidal potential development independently from selected wave groups. We used the HW95 tidal potential development (Hartmann \& Wenzel 1995). In the ET34-ANA-V60 version, the S1 atmospheric wave is analysed and removed by using an impulse response function between gravimetric and barometric records simultaneously with the leastsquares fit to tides to retrieve the gravimetric factor (Schüller 2015). In the older ETERNA3.4 version, there is no distinction between the tidal wave and the atmospheric thermal effect, so the obtained gravimetric factor for S1 is not comparable with ET34-ANA-V60 software. The obtained gravimetric factor at S1 frequency is hence closer to the $\mathrm{S} 1$ tidal wave, but some error still remains in the removal of the atmospheric thermal wave. A tidal analysis computes gravimetric factors and phases that represent the complex transfer function of the Earth to the external tidal potential of the Moon, Sun and other planets, that is, it provides samples of the transfer function defined in eq. (9).

\section{SYNTHETIC TESTS ON NUTATIONS DATA}

We will now focus on a critical question: the sensitivity of the transfer function parameters to errors in the data. The synthetic tests discussed in this section aim at answering three fundamental questions:

(i) What is the minimal error and uncertainty on the estimates that can be achieved using a Bayesian inversion when using data with no error but realistic uncertainties?

\footnotetext{
${ }^{2}$ http://igets.u-strasbg.fr/data_products.php
} ${ }^{1}$ For example https://cddis.nasa.gov/Data_and_Derived_Products/VLBI/V
LBI_data_holdings.html 


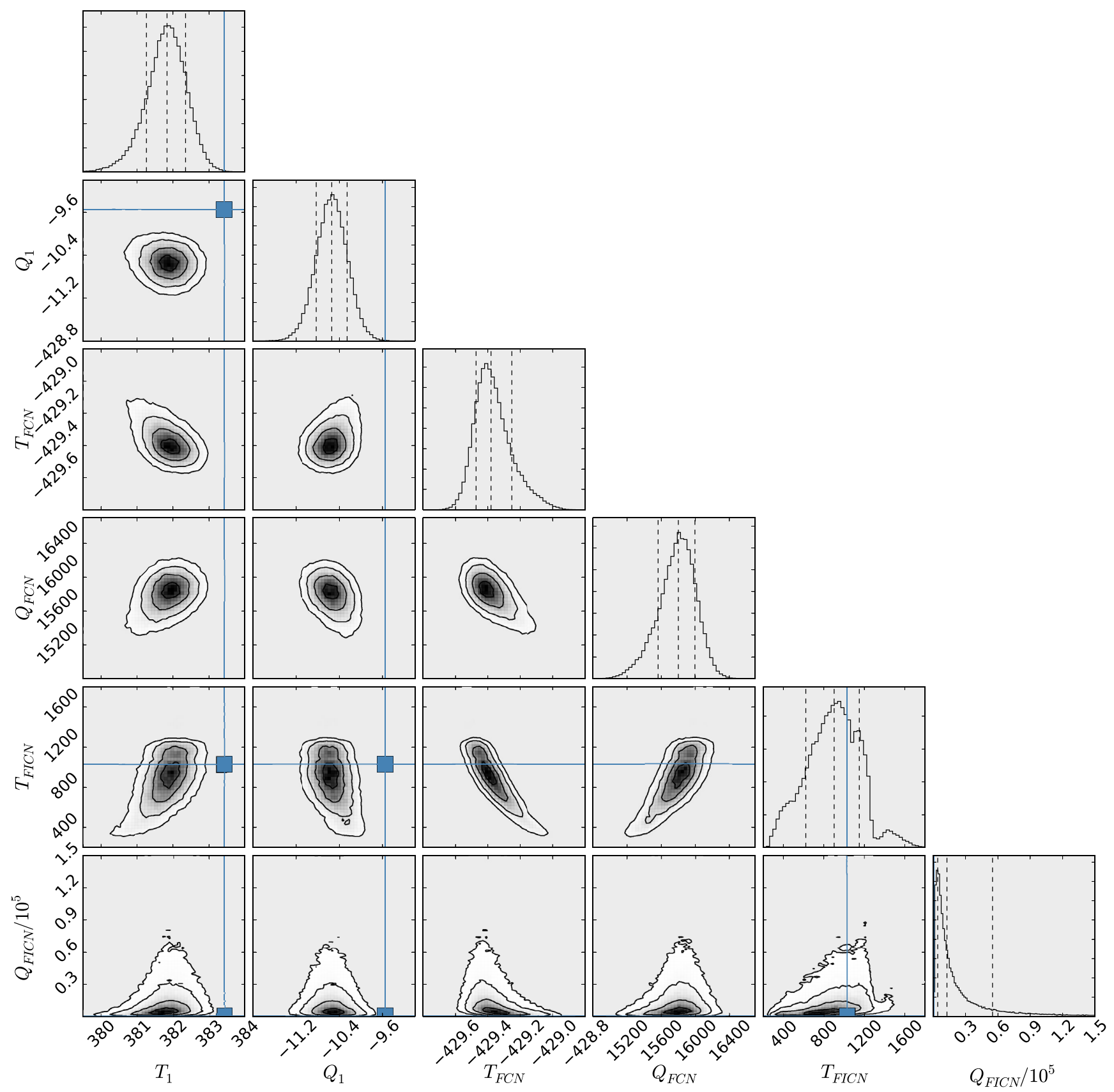

Figure 4. Posterior distributions for the Bayesian inversion of VLBI opa2018b data without atmospheric or oceanic correction. On the diagonal are the distributions for the periods and quality factor of the PM resonance, FCN and FICN. The bi-dimensional distributions (off-diagonal) results from the projection of the sampled models onto a bi-dimensional subset of the parameters space, highlighting the correlations between every possible pair of parameters. Note that the shown parameters (periods and quality factor) were not directly inverted but were computed afterwards from the $N_{\alpha}$ and $\sigma_{\alpha}$ of the resonance formula. The blue lines and squares indicate the values of MHB2000 model parameters. The outer dashed lines, matching the third contour line of the bi-dimensional plots, indicate the 0.16 and 0.84 quantiles, which closely delineate the $1 \sigma$ interval in the Gaussian distributions approximation. The middle dashed line indicates the median. With atmospheric and oceanic corrections applied (not shown), the distributions are closer to Gaussian distributions for $T_{1}, Q_{1}, T_{\mathrm{FCN}}$ and $Q_{\mathrm{FCN}}$ but the multimodality already visible in $T_{\text {FICN }}$ distribution is accentuated, whereas $Q_{\text {FICN }}$ posterior does not notably change.

(ii) What is the influence of noise and/or outliers in the data, and when does the noise level become to high for a successful inversion, especially regarding the FICN?

(iii) Taking into account the fact that the FICN period is not well constrained - assuming it was observed at all, is it possible to retrieve it accurately even if it is much smaller or much larger than the current reference value (of 1030 solar days)?
Answering the first question is required to know how far we can go in the interpretation of the results given in Section 6 but it is also a way to validate our Bayesian algorithm, checking that the credible intervals are always larger than the difference between the estimates and the expected values. The second question is a quantitative way of asking the more fundamental question: can we theoretically see the FICN in real data? We will see that the answer 
is not clear-cut. The third question arises from the recognition that the frequency distribution of the nutations may introduce a bias in favour of certain frequencies for the characterization of the FICN. Here we especially check that even if the FICN lies a bit far away from the closest data points, we can properly retrieve it anyway.

We will only consider the VLBI technique in the following sections because the FCN and FICN parameters are harder to constrain from gravity data anyway (see Section 6.2).

\subsection{Reference model}

A reference synthetic data set without any error $\left(\tilde{\mathrm{T}}=\tilde{\mathrm{T}}_{\mathrm{MHB}}\right.$ and $\left.\tilde{\eta}^{o b s}=\tilde{\mathrm{T}}_{\mathrm{MHB}} \tilde{\eta}_{R}\right)$ is generated using the MHB2000 model with the PM resonance, FCN and FICN terms; the data uncertainties are taken from the opa2018b analysis in order to be as realistic as possible. The ICW being obviously too small to be characterized, it is not included in the model, although we keep calling it MHB2000, instead of 'MHB2000 without ICW'.

The numerical results for the inversion of this error-free synthetic data set are summarized in Table 1 . For all of the periods and quality factors, the target values (used to generate the synthetic data) lie well within the corresponding 68 per cent CIs derived from the Bayesian posteriors. The PM resonance and FCN periods are determined with an uncertainty smaller than half a day and the quality factors are also well constrained. On the contrary, the uncertainties on the FICN parameters reach 100-150 solar days, which is 15 percent of the central estimate. This large uncertainty reflects the uncertainties in the input data combined with the insufficient amount of information they contained: $150 \mathrm{~d}$ is a lower bound on the accuracy of any actual estimate of the FICN period. The median or mean estimate of the FICN period is slightly smaller than 1000 solar days, which is systematically a few percents off of the target value of $1028 \mathrm{~d}$. Taking the central value of a large CI as the best estimate is an hazardous choice, which is why our end results will keep the form of CIs.

\subsection{Influence of the errors}

For the tests with errors, or noise, we could not just make a single test because of the strong sensitivity of the inversion to the distribution of the perturbations in the data. The computing cost of the Bayesian inversion was a limitation, however. We have run 30 inversions with the same level of noise (but different actual error for each data point) and summarized the results in the central part of Table 1 . The errors discussed in this section are sampled from Gaussian distributions with standard deviation equal to the data uncertainty for each data point. Thus, the uncertainties given as input are exact and should not need any scaling.

For each inverted parameter, we give the minimal lower bound and the maximal upper bound of the CIs from every inversion, that is to say the union of all of the individual CIs. We also give the relative error $\Delta \epsilon$ on the CI lower and upper bounds, defined as the difference between the median estimate and the target value, normalized by the Relative Median Absolute Deviation (eq. 15):

$\Delta \epsilon=\frac{\left(P_{\alpha}-P_{\alpha}^{*}\right) / P_{\alpha}^{*}}{R M D}$,

with $P_{\alpha}$ the median estimate of parameter $P$ for eigenmode $\alpha$, and the $\operatorname{star} *$ designating the target value of the inversion (the MHB2000 value for $P_{\alpha}$ ). A value of $|\Delta \epsilon|$ larger than one means that the extent of the CI has been underestimated, excluding either smaller $(\Delta \epsilon<$ $0)$ or larger $(\Delta \epsilon>0)$ values of the estimated parameter.
Among the 30 inversions, we have identified three families, including pathological cases in which the same level of noise could induce very large errors in the estimates. In most cases, however, the inversions are successful and the results are in good agreement with the target values, which are all lying in the CIs: this is the first family of results. It confirms that, even under non-ideal conditions, the Bayesian inversion can still accurately retrieve the transfer function parameters most of the times.

The second family of inversions, about one third of the total, yield results which are also good enough at first sight but some CIs do not actually encompass the corresponding target value $(|\Delta \epsilon|$ larger than one). Such inversions would be indistinguishable from the previous ones if the target values were not known. They reveal that there is no guarantee that the actual value of any model parameter will lie in the estimated CIs, even when the errors on the data are not larger than what we expect in reality. This sensitivity to errors is mostly due to the limited number of observed nutations and their distribution in the frequency band, which is not optimal to constrain the resonances parameters: it originates from an intrinsic lack of information embedded in the data. On a brighter side, though, we could still determine the periods and quality factors with an accuracy within only a few tens of percents for the FICN and even less for the PM resonance and FCN.

The third family, just a few inversions in our tests, yield clearly erroneous results because of the aforementioned underestimation of the error on some nutations which completely bias the model adjustment in their favour. This interpretation is confirmed by the abnormally large scaling factor (up to 100) at the end of the inversion, which illustrates a failed attempt of the inversion algorithm to cope with data points whose uncertainty is too low. Such scenarios are easily detected when inverting real data, by excluding the incriminated data points or by initializing the Markov chains with different starting values. Excluding these obviously inaccurate results, only the first two families (27 valid inversions) have been included in the synthesis of Table 1.

Overall, the PM resonance and FCN parameters are not strongly biased by noise level in agreement with actual data uncertainty. The periods and quality factors of both resonances are always estimated with an accuracy better than a day and a few percents, respectively. Contrary to our expectations, the FICN seems not to be more sensitive to the perturbations in the data.

Additional tests (not shown here) suggest that we can extrapolate the results of this section to much higher level of noise. When we multiply the noise by a factor of up to several tens, all the parameters can still be correctly estimated, in spite of larger CIs. The scaling factor also successfully absorbs the increase in noise level, exactly as expected. Thus, when inverting real data, we expect the Bayesian inversion to give a reliable estimation of the data uncertainty.

\subsection{Other sources of errors}

So far, we have considered that the perturbations in the data were relatively restrained and mostly in agreement with the claimed uncertainties. There are other phenomena, however, that we have not discussed yet but that could also impact the results of the inversion.

The Bayesian inversion is known to be quite robust to outliers. Our complementary synthetic tests have revealed that the outliers either (1) were mostly ignored during the inversion or, on the contrary, (2) biased the results so blatantly that they could be easily identified and removed. For these two reasons, the outliers are a much smaller problem than the nutations with underestimated errors. 


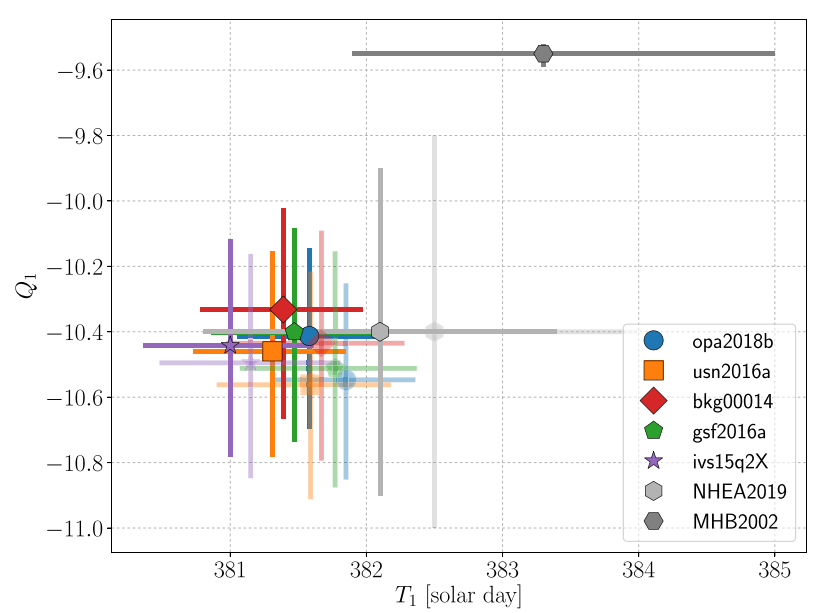

Figure 5. Period (in TRF) and quality factor of the PM resonance estimated from the Bayesian inversions of different data sets from five analysis centres, corrected for atmospheric and oceanic effects (opaque colour symbols). The results from two other studies using least-squares inversion and different data are also shown (grey symbols): MHB2002 for the reference results of Mathews et al. (2002) and NHEA2019 for the recent study of Nurul Huda et al. (2019). The corresponding credible (colour) and confidence (grey) intervals are also displayed. The results without oceanic or atmospheric correction applied are displayed with semitransparent colours for comparison.

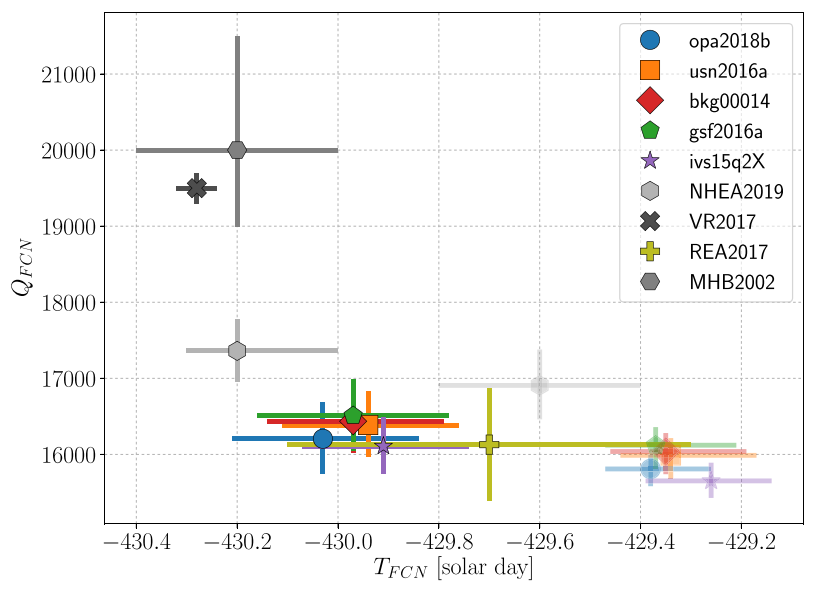

Figure 6. Same as Fig. 5 but for the FCN (period in CRF). REA2017 and VR2017 refer to Rosat et al. (2017) and Vondrák \& Ron (2017) results, respectively.

Regarding the influence of the FICN frequency, we have tested the inversion of synthetic data with noise, as we did in Section 5.2, for a FICN period of 500 and 2000 days (and the same quality factor). Qualitatively, the results strongly suggest that, even if the actual FICN frequency is notably different from the current standard value, we should be able to properly determine it, under the same assumptions on data errors as in Section 5.2, of course.

To conclude these synthetic tests, we look for the maximum level of error we can add to initially error-free synthetic data before the FICN is not detected at all, that is to say, before the Markov chains keep exploring the parameters space without ever reaching stationarity. To do so, we set the data uncertainty at the same value for all nutations (observed nutations normalized by the rigid earth nutations). The detection threshold on the average relative error seems to be close to $10^{-4}$. Below this value, the median estimate can be

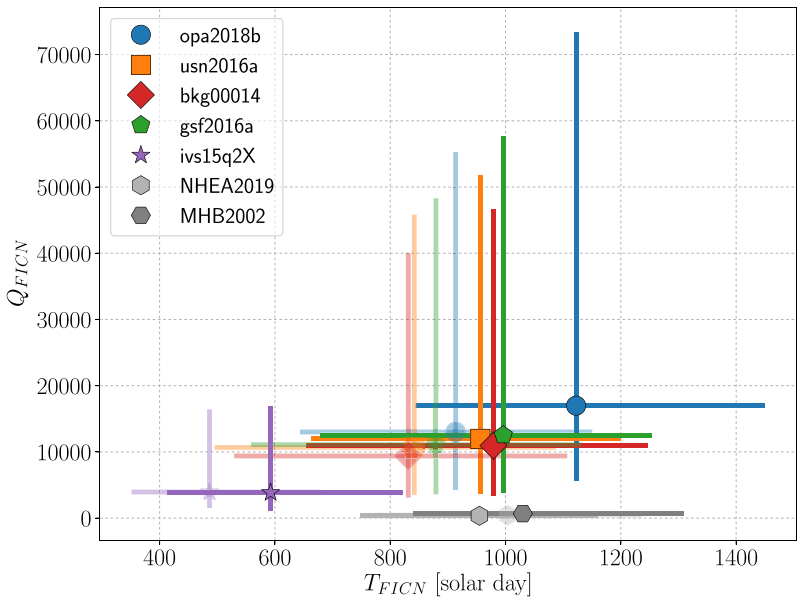

Figure 7. Same as Fig. 5 but for the FICN (period in CRF). Note the difference in scale for the FICN quality factor compared to the previous figures. For this parameter, there seems to be a discrepancy larger than one order of magnitude between our results (colour symbols) and the other studies (grey symbols) but this is not so straightforward. See the text for a correct interpretation of this figure.

off of the target value by a few hundreds days, but the FICN is routinely detected and its parameters can be at least roughly estimated. On the contrary, most of the time, depending on the errors distributions, when the average error is above the aforementioned value, the FICN cannot be consistently detected. As a point of comparison, the median value of the data uncertainties from opa2018b is close to $10^{-3}$ but they strongly vary, from $10^{-5}$ up to 0.5 . Thus, when we did the synthetic tests in Section 5.2, even if the average or median noise could be larger than $10^{-4}$, the errors on data points close to the FICN frequency had actually much smaller errors, which is why the FICN could be properly characterized.

\section{RESULTS OF THE INVERSIONS}

Keeping in mind the results of the synthetic tests, we now move on to the inversion of actual VLBI and gravimetric data. First, we focus on the separated inversions for each technique then, we give the result of the joint inversion.

\subsection{VLBI data inversion}

We invert the amplitudes of the nutations listed on Fig. 2 (without crosses). We have excluded the S1 annual oscillation, known for its perturbation by oceanic and atmospheric effects which are hard to completely correct, as the inversion tends to spuriously identify it with the FICN resonance. For reasons explained in Section 3.2, we have also excluded a few nutations with excessively low uncertainties compared to the other nearby nutations. Overall and after numerous tests, we have thus excluded the following nutations: $\pm 1615,-386,+365$ (S1) and $+346 \mathrm{~d}$. In order to keep consistency, we exclude the same nutations from all five data sets described in Section 4.1 and apply to all of them the corrections for geodesic nutations and non-linear effects given in Mathews et al. (2002, table 7)

Then, we perform two sets of inversions, one without any additional correction, the other with atmospheric and oceanic corrections using Nurul Huda et al. (2019) computations. The results for 
all analysis centres are gathered on Figs 5-7 and the overall estimates with geophysical effects corrected are listed in Table 2. For opa2018b, the posterior distributions for the periods and quality factors are also displayed on Fig. 4 (here without any atmospheric or oceanic correction).

For all resonances, there is a very strong agreement between usn2016a, bkg00014 and gsf2016a for all of the estimated periods and quality factors. In comparison, the results for opa2018b and ivs15q2X are only slightly different for the PM resonance period and FCN period and quality factor but the estimated period is much larger and much smaller, respectively, in the case of the FICN. For this parameter, the CIs for opa2018b and ivs15q2X are not fully compatible with the CIs of the other inversions. We will not expand on the PM resonance here but it is noteworthy that our estimates of $T_{1}$ and $Q_{1}$ are in relatively good agreement with the computed theoretical and inverted values given in (Bizouard et al. 2019) and Nurul Huda et al. (2019), respectively, even if the data were prepared and inverted quite differently in the latter study. For the FCN and FICN, our results differ to varying degrees from both the results of Mathews et al. (2002) and Nurul Huda et al. (2019), with credible intervals and confidence intervals which do not always overlap. Of course, our estimates rely on much more data than what was available at the time of Mathews et al. (2002) study but the recent result of Vondrák \& Ron (2017), which is not compatible with ours, is closer to MHB values.

Figs 5-7 also clearly depict the influence of geophysical effects on the rotational eigenmodes parameters. When correcting for these effects, the PM resonance period decreases by up to $0.3 \mathrm{~d}$ and its quality factor by up to 0.15 . The FCN period increases in absolute value by $0.6 \mathrm{~d}$ and its quality factor increases by a few hundreds $\mathrm{Nu}-$ rul Huda et al. (2019) already noted such variations, although with different values). Similarly, the FICN period increases by 100-200 d when geophysical effects are corrected. In such a case, however, this variation is larger and opposite to what Nurul Huda et al. (2019) obtained.

Even if all parameters seem to be well determined by the Bayesian inversion, the uncertainty on the FICN quality factor should not be overlooked on Fig. 7. What actually happened is that the imaginary part of the FICN frequency could not be properly estimated, leading to a posterior with a peak at zero and a somewhat long tail towards positive values (since we have constrained this parameter to be positive). The maximum of such a distribution is thus very close to zero and very crudely determined. This is a critical issue when computing the quality factor, which is proportional to the inverse of the imaginary part of the frequency. The RMD on $Q_{\text {FICN }}$ reaches the maximum theoretically possible value of 100 percent for all the five Bayesian inversions displayed on Fig. 7, which means, referring to the defining eq. (15), that the variability of the quality factor estimate is as large as the quality factor itself. In other words, the quality factor could have any value between zero and the upper bound of its CI. This is an example where the non-gaussianity of the posterior could lead to a spurious interpretation of the results if no care is taken.

Another parameter for which the posterior distribution differs from the Gaussian distribution, although to a much lesser extent, is the FICN period. As illustrated on Fig. 4, the posterior distribution of $T_{\mathrm{FICN}}$ seems to exhibit some kind of multimodality, with at least one secondary peak at a period larger than the median estimate. This multimodality of the posterior distribution holds true for all analysis centres and for both sets of inversions, with or without geophysical effects corrected.
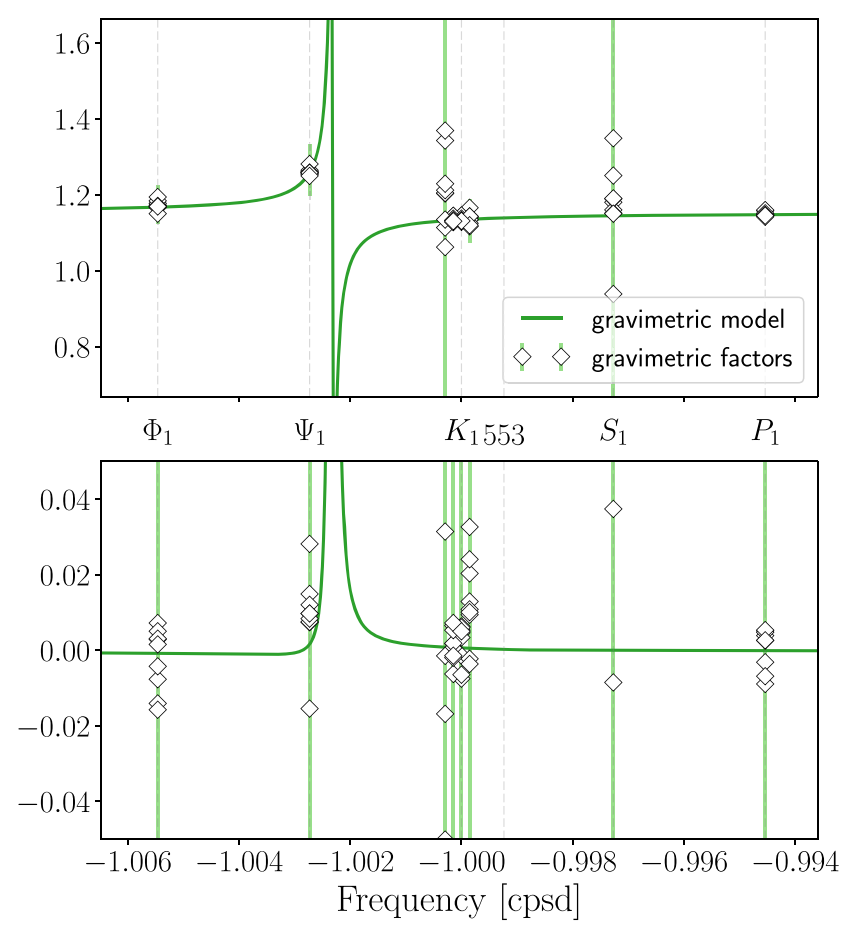

Figure 8. Real (top panel) and imaginary (bottom panel) part of the gravimetric factors in the diurnal frequency band in TRF estimated from the recordings of SGs from nine different gravimetric stations. The transfer functions as defined in the IERS Conventions is depicted in green. For the sake of simplicity, we have chosen not to distinguish the data from each SGs on this plot and to only display a narrow frequency band around the FCN and FICN resonances, similarly to Fig. 1.

Similarly to what we did in the synthetic tests, we have also estimated a scaling factor for the covariance matrix of each data set. For the five analysis centres, the overall data uncertainties were scaled during the inversion by a factor of $1.8-3.0$, revealing a systematic underestimation of the initially given uncertainties.

\subsection{Gravimetric data inversion}

The gravimetric factors have larger errors than their counterparts determined from VLBI data. This may be due to uncorrected instrumental artefacts but this is also a consequence of the high sensitivity of gravimetric measurements which are affected by a great number of geophysical phenomena. Thus, the Bayesian inversion of the FCN and FICN periods and quality factors from gravimetric data requires prior distributions that are more restrictive. Practically, whereas the only constraint on the FCN and FICN frequencies was on the sign of the parameters in the case of VLBI data inversion, we now only allow both estimated periods to be a few hundreds of days off of Mathews et al. (2002) values. Doing so, we notably reduce the size of the parameters space that has to be explored by the Markov chains but we might slightly bias the results of the inversion as an undesired side effect. Even though, this approach is not more prone to biasing the results than what is routinely done with least-squares inversions where the prior values must be chosen very close to the standard reference values to achieve convergence.

All of the gravimetric factors estimated from gravity measurements at nine stations and plotted on Fig. 8 are simultaneously 

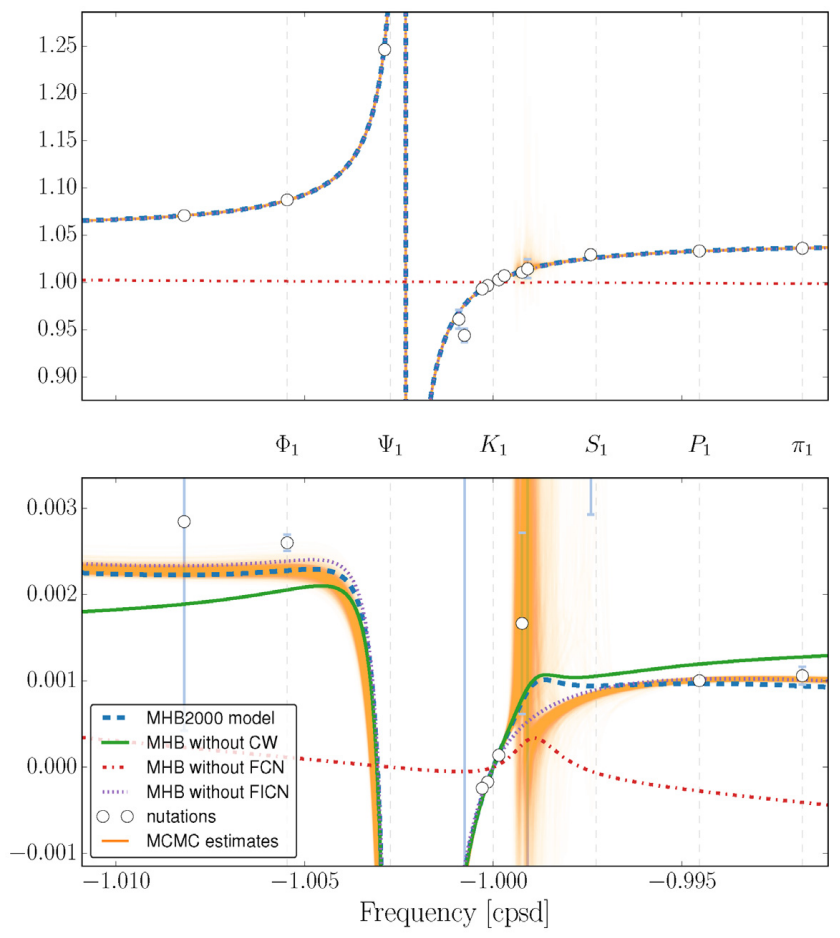

Figure 9. Real (top panel) and imaginary (bottom panel) part of the nutations and different transfer functions in the diurnal frequency band in TRF. The transfer functions MHB2000 as defined in the IERS Conventions is depicted in dashed blue line. One thousand models sampled by the MCMC are plotted in orange lines; they are resampled from the posterior distributions illustrated on Fig. 4. The other transfer functions are computed from the MHB2000 by removing (a) the PM resonance (which is noted CW here) or (b) the FCN or (c) the FICN. These are exclusive "or": for each model, only one of the three resonances is missing. The model without the PM resonance is indistinguishable from the MHB2000 model for the real part; it would completely hide the samples, which is why it is not displayed on the top plot.

inverted. We could have computed the weighted mean of the gravimetric factors across all gravimetric stations for each tidal wave, similarly to what was done in Rosat et al. (2017), but the Bayesian approach exempts us from doing such data combination beforehand. The results of the inversion of gravimetric data are also summarized in Table 2. For the FCN, they are in agreement with the VLBI technique but exhibit a much larger uncertainty, especially for the FCN quality factor which is very roughly determined. Regarding the FICN, the posterior distributions displayed in Rosat et al. (2017) already suggested that the FICN parameters were very poorly constrained by gravimetric data. Indeed, we find a CI for the period which is in good agreement with the VLBI results, although larger, but the quality factor is surprisingly small with an estimated value in the 50-120 range. Considering that from our first Bayesian inversion attempts - without the size reduction of the parameters space described in previous paragraph - we could not even determine the sign of the real part of the FICN resonance amplitude, a conservative conclusion would be to admit that the FICN remains barely visible in gravimetric measurements. As a result, even if the Bayesian inversion can yield a plausible period value for the FICN when restrictive priors are chosen, we cannot guarantee a reliable detection of the FICN in gravity measurements considering the size of the CI for its period and the abnormally well-constrained and small value of the associated quality factor.
The estimation of a scaling factor on the uncertainties, similarly to what we did for the VLBI technique, reveals that the uncertainties on the gravimetric factors are adjusted by a factor of three during the inversion, suggesting that the initial uncertainties were once again underestimated.

\subsection{Joint inversion}

The study of Rosat \& Lambert (2009) is the first attempt to jointly invert VLBI and gravimetric data to estimate the parameters of the FCN resonance. We will now extend their work to also include the FICN and reconsider the interest of the Bayesian joint inversion. The common geophysical parameters in the joint inversion are the complex frequencies $\tilde{\sigma}_{\alpha}$ whereas the amplitudes $\tilde{N}_{\alpha}$ and $\tilde{N}_{\alpha}^{g}$ are inverted separately.

The results of the joint inversion are given in Table 2 for opa2018b (the other data sets yield similar results), in comparison to the results of the separated inversions. The most prominent observation is that the CIs from the joint inversion are overall very similar to the CIs from the inversion of VLBI data exclusively as already noted by Rosat \& Lambert (2009). There is no difference in the PM resonance and FCN period or quality factor CIs that we would consider conclusive; this was expected considering the much more accurate results obtained from VLBI data compared with gravimetric data. The FICN is an exception in the case of opa2018b, with a CI from the joint inversion much more similar to what we obtain for other analysis centres (e.g. usn2016a) compare to the case of VLBI data inversion alone (see Fig. 7). In other words, the addition of gravimetric data to the opa2018b nutations tends to shift the FICN period $\mathrm{CI}$ as a whole towards smaller periods, cancelling the disagreement with other VLBI analysis centres. This interpretation should be qualified, however. We have already discussed in Sections 5.1 and 6.1 the hazardous interpretation of large CIs and we have underlined in Section 6.2 the possible multimodality of the FICN period posterior distribution, as well as the dubious value of the associated quality factor. Thus, we should remember that, even when using Bayesian methods, the interpretation of the estimate of a loosely constrain parameter remains a difficult problem. In addition, this change in the FICN period when using opa2018b in the joint inversion is not observed when using ivs15qX2 data set instead. In the latter case, the results of the joint inversion are not notably different from the results of the VLBI data-only inversion.

\subsection{Model testing}

Even if most authors have adjusted the PM, FCN and FICN resonances, and sometimes even the ICW, without much discussion, we think that assessing the relevance of our FICN parameters estimation is necessary.

Focusing on the VLBI technique, Fig. 9 depicts the same nutations data and MHB2000 model as Fig. 1 but three more models are plotted: (a) MHB2000 without the PM resonance, (b) MHB2000 without the FCN and (c) MHB2000 without the FICN. They illustrate the respective importance of the resonances in the nutations due to the mantle, outer core and inner core rotation, respectively. The PM and FCN resonances are readily visible, both as a shift in amplitude impacting all the nearly-diurnal nutations and as a more prominent peak around the FCN frequency for the FCN. This qualitatively illustrates why we can determine quite accurately the period and quality factor of these resonances. On the contrary, the 
difference between the original MHB2000 model and model (c) is blatantly small compared to the data uncertainties and actual errors.

After removing the FICN from the model and inverting the VLBI data again, we have compared the remaining estimated parameters and the residuals (wRMSE) between this simplified model (without the FICN) and the one used in Section 6.1 (with the FICN). The residuals are 10-20 percent larger when the FICN is not included in the model, suggesting that the fit is slightly improved when a resonance is adjusted in the prograde diurnal band. Most of the parameters estimates also change at least slightly, if not substantially. The scaling factor notably is four times larger when the FICN is excluded from the inverted model. Thus, even if the FICN seems superfluous at first sight to explain the data, it seems that we are sensitive to its associated resonance anyway.

Considering how the scaling factor increases when the model used in the inversion is not complete, it can also be interpreted as the revelation of an incomplete theory. The uncertainty on the model is not the uncertainty resulting from the uncertainty on the model parameter, it comes from the approximations made in the theoretical developments. Practically, it is equivalent to consider that there are some uncorrected errors in the data or to consider that the model has a small unknown component that could explain to some extent a discordance with the data. That is why, in the present work, we have chosen to re-estimate the data uncertainty while considering that the theoretical model, only depending on the PM, FCN and FICN resonance parameters, is perfect.

\section{DISCUSSION}

By including the results of numerous synthetic tests in Section 5, we have defended the idea that a systematic assessment of the influence of errors in both the data and their uncertainty was a necessity before we could make any reliable geophysical interpretation. Thus, we shall discuss the results of VLBI and gravimetric data inversions keeping in mind the conclusions of Section 5.

The inversion of nutations computed from different data sources has revealed a large consistency for most inverted parameters, along with an overall good agreement between the Bayesian CIs. Both interpretations of such a good consistency could be enlightening: either (1) most analysis centres introduce the same errors or biases in their analysis and this work is a prominent illustration of such a problem or (2) there is no significant bias and our estimates are robust. Obviously, the second option is preferable, but the reality might be midway between both alternatives. The possibility of systematic biases in nutations estimates cannot be easily ruled out as the VLBI analysis centres largely process the same raw data with a limited number of widespread softwares and similar procedures (even for ivs15q2X which is only partly an exception). As such, their products are far from being independent. This is why gravimetric data, as an independent data set, are still so valuable in rotational eigenmodes studies, in spite of their larger errors and uncertainty.

The FCN period we find from the VLBI data or joint inversion, approximately -430.2 to -429.8 solar days, is not fully compatible with the results of other studies. Rosat et al. (2017) give a lower bound at $-430.1 \mathrm{~d}$, Mathews et al. (2002) and Nurul Huda et al. (2019) provide estimates ranging from -430.4 to $-430.0 \mathrm{~d}$ and Vondrák \& Ron (2017) suggest an even larger period (in absolute value), at $-430.28 \pm 0.04 \mathrm{~d}$. In most of these works, the FCN quality factor is significantly larger than our values as well.

The difference between our estimates and others may be due to a spurious side-effect of our data selection but, conversely, it might also be the FCN period found in other studies that was biased by the nutations we have chosen to remove. We may ask whether there is a larger undesired bias when keeping abnormal data points or when removing them; such a classic problem is not easily solved. In any case, if the eigenmodes parameters are so sensitive to such a limited subset of data points, it is another reason why we strongly recommend to improve the upstream estimation of data uncertainty. Indeed, most of the nutations we had to exclude were biasing the inversion because of their too large uncorrected errors relative to their estimated uncertainty. Once again, note that the scaling factor we adjust in all inversions cannot efficiently counterbalance very underestimated uncertainty on a small number of data points since this factor is applied on the entire covariance matrix.

Of course, there are many other differences between this work and previous studies, whether it be on the inverted data sets, inversion methods or the corrections applied to the data. For example, we did not consider the possible influence of geomagnetic jerks contrary to Vondrák \& Ron (2017). Yet they found a slightly smaller FCN period (in absolute value) when taking them into account, thus suggesting that such an effect does not necessarily explain the difference we observe between our result and theirs because it might have increased the discrepancy, had we corrected for it. Zhou et al. (2016) provide their own estimate of the FCN period and list several other estimates from other authors who used different data sets, different methods and different corrections. The discrepancies between these results is actually much larger than the differences which are visible on Fig. 6 and that we have been discussing so far. In addition, Lambert \& Dehant (2007) have shown that the intrinsic variation of the FCN period with time could make it change by more than a day across several years. Thus, it seems reasonable to consider that differences smaller than $1 \mathrm{~d}$ should not be prematurely interpreted unless they occur between studies relying on identical data sets and applying similar inversion methods.

Finally, regarding the FICN, the main question we were aiming to address was about its actual detection. Since the work of Mathews et al. (2002) many studies have often been inconclusive by failing to properly characterize the FICN. Considering the reasonably good consistency of our results on Fig. 7 and the CIs and RMDs for the FICN period in Table 2, it seems that, indeed, we can detect the influence of the solid inner core on the Earth nutations. As to whether or not we can accurately estimate the FICN period, the answer is not clear-cut. There are discrepancies between different estimates, but no straightforward explanation. The relatively better agreement between Nurul Huda et al. (2019) and Mathews et al. (2002) for the quality factor suggests that the use of longer and more accurate VLBI data is not a good enough explanation. Thus, the differences might stem from our Bayesian approach which differs from the least-squares method used in both previous studies. The multimodal posterior we found for the FICN period might explain why the least-squares method yields different results even if further investigations would be required to draw a definitive conclusion. That being said, we should probably not worry too much about these differences until we can notably decrease the uncertainties on the FICN parameters estimates.

\section{CONCLUSION}

One of the main goal of this work was to assess the interest of a joint Bayesian inversion of VLBI and gravimetric data to estimate the FCN and FICN parameters. We have shown that such approach 
could yield satisfactory results, benefiting from the most informative data in the joint data set. Had we only rely on separated inversions, we could have only concluded that both techniques yielded contradictory results for the FICN quality factor, for example. It will be probably necessary to improve the correction and analysis of gravimetric data, however, before the joint inversion can offer a significant added value compared to the inversion of VLBI data only.

We also endeavoured to confirm past observations of the resonance associated with the FICN and to provide a reliable credible interval for its period. Our conservative estimates range from 500 to 1300 solar days, which is admittedly not a better level of accuracy than what Mathews et al. (2002) obtained over 15 yr ago using a least-squares inversion and much shorter VLBI time-series. This suggests that, either their uncertainties were strongly underestimated or we have yet to properly model and correct non-negligible systematic errors in the data. The failure of the Bayesian inversion to yield a well-constrained estimate of the FICN quality factor tends to demonstrate that both aforementioned hypotheses are probably valid.

In the theoretical frame of the angular momentum description used in the MHB2000 model, the primary reason for such a large FICN period is the couplings at the ICB and CMB, especially the electromagnetic couplings. Even so, recent studies, mostly relying on a linear momentum description, have raised concerns about the validity of previous reference theories and suggested that the angular momentum approach through Liouville's equations was not sufficient to fully describe all nutational modes (Rogister \& Valette 2009; Seyed-Mahmoud et al. 2017). Rochester et al. (2014) and Crossley \& Rochester (2014) have obtained yet different results, especially for the FICN, proving that further theoretical work has still to be done. Therefore, we will refrain for now from interpreting our results in terms of electromagnetic coupling or, for that matter, in terms of any such well-defined geophysical process.

\section{ACKNOWLEDGEMENTS}

We would like to thank all the VLBI and gravimetry communities for acquiring, preparing and sharing the data processed and inverted in this study. We thank two anonymous reviewers for their valuable comments which have contributed to improve the original manuscript and some aspects of this study. This work was funded by CNES (Centre National d'Études Spatiales).

\section{REFERENCES}

Barnes, R.T.H., Hide, R.,F.R.S., White, A.A. \& Wilson, C.A., 1983. Atmospheric angular momentum fluctuations, length-of-day changes and polar motion, Proc. R. Soc. Lond., A, 387(1792), 31-73.

Bizouard, C., Nurul Huda, I., Ziegler, Y. \& Lambert, S., 2019. Frequency dependence of the polar motion resonance, Geophys. J. Int., 220(2), 753758.

Boy J.-P., Barriot J.-P., Förste C., Voigt C. \& Wziontek H., 2020. Achievements of the First 4 Years of the International Geodynamics and Earth Tide Service (IGETS) 2015-2019, International Association of Geodesy Symposia.

Brzeziński, A., Bizouard, C. \& Petrov, S.D., 2002. Influence of the atmosphere on earth rotation: what new can be learned from the recent atmospheric angular momentum estimates?, Surv. Geophys., 23( 1), 33-69.

Buffett, B.A., Mathews, P.M. \& Herring, T.A., 2002. Modeling of nutation and precession: effects of electromagnetic coupling, J. geophys. Res., 107, ETG 5-1-ETG 5-14.
Busse, F.H., 1970. The dynamical coupling between inner core and mantle of the earth and the 24-year libration of the pole, in Earthquake Displacement Fields and the Rotation of the Earth, pp. 88-98, Springer Science + Business Media.

Chao, B.F. \& Hsieh, Y., 2015. The earth's free core nutation: formulation of dynamics and estimation of eigenperiod from the very-long-baseline interferometry data, Earth planet. Sci. Lett., 432, 483-492.

Crossley, D.J. \& Rochester, M.G., 2014. A new description of Earth's wobble modes using Clairaut coordinates 2: results and inferences on the core mode spectrum, Geophys. J. Int., 198(3), 1890-1905.

Cummins, P., Wahr, J.M., Agnew, D.C. \& Tamura, Y., 1991. Constraining core undertones using stacked IDA gravity records, Geophys. J. Int., 106(1), 189-198.

Dehant, V., Defraigne, P. \& Wahr, J.M., 1999. Tides for a convective earth, J. geophys. Res., 104( B1), 1035-1058.

Dehant, V., de Viron, O. \& Greff-Lefftz, M., 2005. Atmospheric and oceanic excitation of the rotation of a three-layer earth, Astron. Astrophys., 438, 1149-1161.

Dehant, V., Hinderer, J., Legros, H. \& Lefftz, M., 1993. Analytical approach to the computation of the earth, the outer core and the inner core rotational motions, Phys. Earth planet. Inter., 76(3-4), 259-282.

de Vries, D. \& Wahr, J.M., 1991. The effects of the solid inner core and nonhydrostatic structure on the earth's forced nutations and earth tides, $J$. geophys. Res., 96( B5), 8275.

Ding, H. \& Chao, B.F., 2017. Solid pole tide in global GPS and superconducting gravimeter observations: signal retrieval and inference for mantle anelasticity, Earth planet. Sci. Lett., 459, 244-251.

Florsch, N. \& Hinderer, J., 2000. Bayesian estimation of the free core nutation parameters from the analysis of precise tidal gravity data, Phys. Earth planet. Inter., 117, 21-35.

Gattano, C., Lambert, S.B. \& Bizouard, C., 2016. Observation of the earth's nutation by the VLBI: how accurate is the geophysical signal, J. Geod., 91(7), 849-856.

Greff-Lefftz, M., Legros, H. \& Dehant, V., 2000. Influence of the inner core viscosity on the rotational eigenmodes of the earth, Phys. Earth planet. Inter., 122(3-4), 187-204.

Gwinn, C.R., Herring, T.A. \& Shapiro, I.I., 1986. Geodesy by radio interferometry: studies of the forced nutations of the earth - 2. Interpretation, J. geophys. Res., 91, 4755.

Hartmann, T. \& Wenzel, H.-G., 1995. The HW95 tidal potential catalogue, Geophys. Res. Lett., 22, 3553-3556.

Herring, T.A., Gwinn, C.R. \& Shapiro, I.I., 1986. Geodesy by radio interferometry: studies of the forced nutations of the earth - 1. Data analysis, J. geophys. Res., 91, 4745-4754.

Herring, T.A., Mathews, P.M. \& Buffett, B.A., 2002. Modeling of nutationprecession: very long baseline interferometry results, J. geophys. Res., 107(B4), ETG 4-1-ETG 4-12.

Hinderer, J., Crossley, D. \& Warburton, R.J., 2007. Gravimetric methodssuperconducting gravity meters, in Treatise on Geophysics - Volume 3: Geodesy, pp. 65-122, eds Herring, T. \& Schubert, G., Elsevier.

Hopkins, W., 1839. Researches in physical geology: on the phenomena of precession and nutation, assuming the fluidity of the interior of the earth, Phil. Trans. R. Soc. Lond., 129, 381-423.

Hough, S.S., 1895. The oscillations of a rotating ellipsoidal shell containing fluid, Phil. Trans. R. Soc. Lond., A, 186, 469-506.

Koot, L., Dumberry, M., Rivoldini, A., de Viron, O. \& Dehant, V., 2010. Constraints on the coupling at the core-mantle and inner core boundaries inferred from nutation observations, Geophys. J. Int., 182(3), 1279-1294.

Koot, L., Rivoldini, A., de Viron, O. \& Dehant, V., 2008. Estimation of earth interior parameters from a Bayesian inversion of very long baseline interferometry nutation time series, J. geophys. Res., 113, B08414.

Lambert, S.B. \& Dehant, V., 2007. The earth's core parameters as seen by the VLBI, Astron. Astrophys., 469(2), 777-781.

Lambert, S.B., Rosat, S., Cui, X., Rogister, Y. \& Bizouard, C., 2012. A search for the free inner core nutation in VLBI data, in Proceedings of the 7th IVS General Meeting, pp. 370-374. 
Lecolazet, R. \& Steinmetz, L., 1974. Sur les ondes diurnes de la marée gravimétrique observée à strasbourg, Compt. Rend. Acad. Sci., B, 278, 295-297.

Legros, H., Hinderer, J., Lefftz, M. \& Dehant, V., 1993. The influence of the solid inner core on gravity changes and spatial nutations induced by luni-solar tides and surface loading, Phys. Earth planet. Inter., 76(3-4), 283-315.

Mathews, P.M., 2001. Love numbers and gravimetric factor for diurnal tides, J. Geod. Soc. Jpn., 47(1), 231-236.

Mathews, P.M., Buffett, B.A., Herring, T.A. \& Shapiro, I.I., 1991a. Forced nutations of the Earth: influence of inner core dynamics. 1 - Theory, $J$. geophys. Res., 96(B5), 8219-8242.

Mathews, P.M., Buffett, B.A., Herring, T.A. \& Shapiro, I.I., 1991b. Forced nutations of the earth: Influence of inner core dynamics: 2. Numerical results and comparisons, J. geophys. Res., 96(B5), 8243.

Mathews, P.M., Herring, T.A. \& Buffett, B.A., 2002. Modeling of nutation and precession: new nutation series for nonrigid earth and insights into the earth's interior, J. geophys. Res., 107(B4), 2068.

Melchior, P., 1980. For a clear terminology in the polar motion investigations, in Nutation and the Earth's Rotation, pp. 17-21, Springer Netherlands.

Melchior, P. \& Georis, B., 1968. Earth tides, precession-nutation and the secular retardation of earth's rotation, Phys. Earth planet. Inter., 1(4), $267-287$.

Neuberg, J., Hinderer, J. \& Zurn, W., 1987. Stacking gravity tide observations in central Europe for the retrieval of the complex eigenfrequency of the nearly diurnal free-wobble, Geophys. J. Int., 91(3), 853-868.

Nothnagel, A., Artz, T., Behrend, D. \& Malkin, Z., 2016. International VLBI service for geodesy and astrometry, J. Geod., 91( 7), 711-721.

Nurul Huda, I., Bizouard, C., Ziegler, Y. \& Lambert, S., 2019. Nutation terms adjustment and implication for the earth rotation resonance parameters, Geophys. J. Int., 220(2), 759-767.

Patil, A., Huard, D. \& Fonnesbeck, C., 2010. PyMC: Bayesian stochastic modelling in Python, J. Stat. Softw., 35(4), 1-81.

Petit, G. \& Luzum, B., 2010. IERS Conventions (2010), techreport, IERS Convention Centre.

Poincaré, H., 1910. Sur la précession des corps déformables, Bull. Astron., I, XXVII, 321-356.

Rochester, M.G., 1973. The earth's rotation, EOS, Trans. Am. Geophys. Un, 54 (8), 769-780.

Rochester, M.G., Crossley, D.J. \& Zhang, Y.L., 2014. A new description of Earth's wobblemodes using Clairaut coordinates: 1. Theory, Geophys. J. Int., 198( 3), 1848-1877.

Rochester, M.G., Jensen, O.G. \& Smylie, D.E., 1974. A search for the earth's nearly diurnal free wobble, Geophys. J. R. astr. Soc., 38(2), 349-363.

Rogister, Y. \& Valette, B., 2009. Influence of liquid core dynamics on rotational modes, Geophys. J. Int., 176(2), 368-388.

Roosbeek, F. \& Dehant, V., 1998. RDAN97: an analytical development of rigid earth nutation series using the torque approach, Celest. Mech. Dyn. Astron., 70, 215-253.

Rosat, S., Calvo, M. \& Lambert, S., 2016. Detailed analysis of diurnal tides and associated space nutation in the search of the free inner core nutation resonance, in International Association of Geodesy Symposia, Springer Science + Business Media.
Rosat, S., Florsch, N., Hinderer, J. \& Llubes, M., 2009. Estimation of the free core nutation parameters from SG data: sensitivity study and comparative analysis using linearized least-squares and Bayesian methods, J. Geodyn., 48, 331-339.

Rosat, S. \& Lambert, S.B., 2009. Free core nutation resonance parameters from VLBI and superconducting gravimeter data, Astron. Astrophys., 503, 287-291.

Rosat, S., Lambert, S.B., Gattano, C. \& Calvo, M., 2017. Earth's core and inner core resonances from analysis of VLBI nutation and superconducting gravimeter data, Geophys. J. Int., 208, 211-220.

Rousseeuw, P.J. \& Croux, C., 1993. Alternatives to the median absolute deviation, J. Am. Stat. Assoc., 88(424), 1273-1283.

Sasao, T., Okubo, S. \& Saito, M., 1980. A simple theory on the dynamical effects of a stratified fluid core upon nutational motion of the earth, in Nutation and the Earth's Rotation, vol. 78 of IAU Symposium, p. 165.

Schüller, K., 2015. Theoretical basis for earth tide analysis with the new ETERNA34-ANA-V4.0 program, Bull. Inf. Marées Terr., 149, 12024 12062.

Seyed-Mahmoud, B., Rochester, M.G. \& Rogers, C.M., 2017. Truncation effects in computing free wobble/nutation modes explored using a simple earth model, Geophys. J. Int., 209(3), 1455-1461.

Sludskii, F., 1896. De la rotation de la terre supposée fluide á son interieur, Bull. Soc. Natur. Moscou, 9, 285-318.

Souchay, J., Loysel, B., Kinoshita, H. \& Folgueira, M., 1999. Corrections and new developments in rigid earth nutation theory. III. Final tables "REN-2000" including crossed-nutation and spin-orbit coupling effects, Astron. Astrophys., 135, 111-131.

Toomre, A., 1974. On the 'nearly diurnal wobble' of the earth, Geophys. J. Int., 38(2), 335-348.

VanderPlas, J., 2014. Frequentism and Bayesianism: a Python-driven primer, Proceedings of the 13th Python in Science Conference, 91-99, van, der Walt S. \& Bergstra, J.

Vondrák, J. \& Ron, C., 2014. Geophysical excitation of nutation - comparison of different models, Acta Geodyn. Geomater, 11 (3), 193-200.

Vondrák, J. \& Ron, C., 2017. New method for determining free core nutation parameters, considering geophysical effects, Astron. Astrophys., 604, A56.

Wahr, J.M., 1981a. A normal mode expansion for the forced response of a rotating earth, Geophys. J. R. astr. Soc., 64, 651-675.

Wahr, J.M., 1981b. Body tides on an elliptical, rotating, elastic and oceanless earth, Geophys. J. R. astr. Soc., 64, 677-703.

Wahr, J.M., 1981c. The forced nutations of an elliptical, rotating, elastic and oceanless earth, Geophys. J. R. astr. Soc., 64, 705-727.

Wenzel, H.-G., 1996. The Nanogal software: earth tide data processing package ETERNA 3.30, Bull. Inf. Marées Terr., 124, 9425-9439.

Zhou, Y., Zhu, Q., Salstein, D.A., Xu, X., Shi, S. \& Liao, X., 2016. Estimation of the free core nutation period by the sliding-window complex leastsquares fit method, Adv. Space Res., 57( 10), 2136-2140.

Zhu, P., Rivoldini, A., Koot, L. \& Dehant, V., 2017. Basic earth's parameters as estimated from VLBI observations, Geod. Geodyn., 8, 427-432.

Ziegler, Y., Hinderer, J., Rogister, Y. \& Rosat, S., 2016. Estimation of the gravimetric pole tide by stacking long time-series of GGP superconducting gravimeters, Geophys. J. Int., 205, 77-88. 